\title{
Large-scale conformational changes of FhaC provide insights into the
}

\section{two-partner secretion mechanism}

4 G. Sicoli ${ }^{1 *}$, A. Konijnenberg ${ }^{2 *}$, J. Guerin $^{3 \$}$, S. Hessmann ${ }^{4,5}$, E. Del Nero ${ }^{4,5}$, O. Hernandez-Alba ${ }^{4,5}$, 5 S. Lecher ${ }^{3}$, G. Rouaut ${ }^{6}$, L. Müggenburg ${ }^{6 \$}$, H. Vezin ${ }^{1}$, S. Cianférani ${ }^{4,5}$, F. Sobott ${ }^{2,7}$, R. Schneider ${ }^{6 \$ £}$, F. Jacob-Dubuisson ${ }^{3 £}$

$8{ }^{1}$ Laboratoire Avancé de Spectroscopie pour les Interactions, la Réactivité et l'Environnement (LASIRE), UMR CNRS

9 8516, Université de Lille, Avenue Paul Langevin - C4, F-59655 Villeneuve d'Ascq Cedex, France

${ }^{2}$ BAMS Research group, University of Antwerp, Groenenborgerlaan 171, 2020 Antwerp, Belgium

${ }^{3}$ CNRS, INSERM, Institut Pasteur de Lille, U1019-UMR9017-CIIL-Center for Infection and Immunity of Lille, F59000, Lille, France Strasbourg 67000, France

${ }^{5}$ Infrastructure Nationale de Protéomique ProFI - FR2048, 67087 Strasbourg, France 59650 Villeneuve d'Ascq, France

${ }^{7}$ Astbury Centre for Structural Molecular Biology and the School of Molecular and Cellular Biology, University of

* These authors contributed equally.

$22 £$ These authors contributed equally. For correspondence: robert.schneider@univ-lille.fr;

23 francoise.jacob@ibl.cnrs.fr

24 current addresses: AK, Thermo Fisher Scientific, Zwaanstraat 31 G/H, Building TR, 5651 Eindhoven, The 25 Netherlands; JG, Calixar, 60 avenue Rockefeller, 69008 Lyon, France; LM, Institut für Organische Chemie, 26 Gottfried Wilhelm Leibniz Universität Hannover, Schneiderberg 1B, DE-30167 Hannover, Germany; RS, Bruker 27 Switzerland AG, Industriestrasse 26, CH-8117 Fällanden, Switzerland. 


\section{Abstract}

The Two-Partner secretion pathway mediates protein transport across the outer membrane of Gram-negative bacteria. TpsB transporters belong to the Omp85 superfamily, whose members catalyze protein insertion into, or translocation across membranes without external energy sources.

They are composed of a transmembrane $\beta$ barrel preceded by two periplasmic POTRA domains that bind the incoming protein substrate. Here we used an integrative approach combining in vivo assays, mass spectrometry, nuclear magnetic resonance and electron paramagnetic resonance techniques suitable to detect minor states in heterogeneous populations, to explore transient conformers of the TpsB transporter FhaC. This revealed substantial, spontaneous conformational changes with a portion of the POTRA2 domain coming close to the lipid bilayer and surface loops. Specifically, the amphipathic $\beta$ hairpin immediately preceding the first barrel strand can insert into the $\beta$ barrel. We propose that these motions enlarge the channel and hoist the substrate into it for secretion. An anchor region at the interface of the $\beta$ barrel and the POTRA2 domain stabilizes the transporter in the course of secretion. Our data propose a solution to the conundrum how these transporters mediate protein secretion without the need for cofactors, by utilizing intrinsic protein dynamics.

\section{Introduction}

The Two-Partner Secretion (TPS) pathway is dedicated to the export of large proteins notably serving as virulence factors (Guerin et al., 2017). The TpsB transporters are transmembrane $\beta$ barrel proteins that secrete their substrates, collectively called TpsA proteins, across the outer membrane of various Gram-negative bacteria. They belong to the ubiquitous Omp85 superfamily whose members mediate protein insertion into, or translocation across membranes of bacteria and 
51 eukaryotic organelles, and which includes the essential bacterial BamA transporters (Heinz \&

52 Lithgow, 2014; Knowles et al., 2009; Noinaj et al., 2017). The FhaB/FhaC pair of Bordetella

53 pertussis is a model TPS system, in which the FhaC transporter mediates the translocation of the

54 adhesin FhaB across the outer membrane (Fan et al., 2012).

55 Omp85 transporters are composed of N-terminal POTRA (polypeptide transport associated)

56 domains - two in the case of TpsB transporters - followed by a 16-stranded transmembrane $\beta$ barrel,

57 which for FhaC is the FhaB translocation pore (Baud et al., 2014). The POTRA domains mediate

58 protein-protein interactions in the periplasm, and notably recognition of client proteins (Delattre

59 et al., 2011). Another hallmark feature of the Omp85 superfamily is the extracellular loop L6 that

60

61

62

63

64

65

66

67

68

69

70

71

72

73 folds back inside the barrel and harbors a conserved motif at its tip forming a salt bridge interaction with a specific motif of the inner $\beta$-barrel wall (Gu et al., 2016; Maier et al., 2015; Noinaj et al., 2013).

A specific feature of TpsB transporters is an $\mathrm{N}$-terminal $\alpha$ helix called $\mathrm{H} 1$ that plugs the $\beta$ barrel (Clantin et al., 2007; Guerin et al., 2014; Guerin et al., 2020; Maier et al., 2015) (Fig. 1A, B). An extended linker follows $\mathrm{H} 1$ and joins it to the POTRA1 domain in the periplasm. Recently, the Xray structures of the $\mathrm{TpsB}$ transporters $\mathrm{CdiB}^{\mathrm{Ab}}$ and $\mathrm{CdiB}^{\mathrm{Ec}}$ have shown very similar folds to that of FhaC, albeit with slightly different positions of H1 in the barrel (Guerin et al., 2020). Both H1 and L6 stabilize the barrel in a closed conformation that most likely corresponds to the resting state of the transporter (Guerin et al., 2020; Maier et al., 2015). The $\beta$ barrel, the L6 loop and the two POTRA domains are essential for transport activity (Clantin et al., 2007).

Omp85 transporters likely function in the absence of ATP or an electrochemical gradient. They appear to be very dynamic and to undergo conformational cycling (Doerner \& Sousa, 2017; Guerin et al., 2020; Hartmann et al., 2018; Iadanza et al., 2020; Renault et al., 2011; Warner et al., 2017). 
74 Lateral opening of the barrel between the first and last anti-parallel $\beta$ strands is a common

75 mechanistic feature of Omp85 transporters, which is involved in their respective functions

76 (Diederichs et al., 2020; Doyle \& Bernstein, 2019; Estrada Mallarino et al., 2015; Guerin et al.,

77 2020; Höhr et al., 2018; Iadanza et al., 2016; Noinaj et al., 2014; Tomasek et al., 2020).

78 The mechanism of two-partner secretion remains poorly understood, but it is known to involve

79 substantial conformational changes of the transporter including exit of $\mathrm{H} 1$ from the $\beta$ barrel and

80

81

82

motions of the L6 loop (Guerin et al., 2014; Guerin et al., 2020; Guerin et al., 2015) (Figure 1B).

The motion of $\mathrm{H} 1$ toward the periplasm is facilitated by conformational changes of flexible regions of the barrel, in particular the first $\beta$-barrel strand B1 and the extracellular loops L1, L2 and L6 (Guerin et al., 2020). Binding of the N-terminal, conserved 'TPS' domain of the substrate protein to the POTRA domains of its transporter also appears to enhance conformational changes (Guerin et al., 2015). How the substrate enters the pore and is progressively hoisted towards the cell surface without backsliding to the periplasm remains unknown, but we hypothesize a mechanism implying yet uncharacterized transient conformations of TpsB transporters. In this work we have explored such FhaC conformers using biophysical techniques suitable to detect minor states in heterogeneous populations. Our data revealed an intrinsic exchange of the POTRA2 domain between several conformations in slow equilibrium, and that these conformational changes are linked to transport activity.

\section{Results}

\section{Effects of freezing the POTRA2 conformation on secretion activity}

We have previously obtained evidence that, in addition to the H1 helix and the L6 loop, the POTRA2 domain also undergoes conformational changes during secretion (Guerin et al., 2015). 
97 To determine which POTRA2 regions must be mobile for secretion, we searched for specific $\mathrm{H}$

98

99

100

101

102

103

104

105

106

107

108

109

110

111

112

113

114

115

116

117

118

bond- or salt bridge-mediated interactions present in the resting conformation (i.e., corresponding to the crystallographic structure) and disrupted them to loosen the structure or conversely replaced them with disulfide (S-S) bonds to limit motions of the corresponding regions. Of note, FhaC is naturally devoid of Cys residues. Residues involved in interactions between the POTRA2 domain and the barrel $\left(\mathrm{Asn}^{245}-\mathrm{Ser}^{157}\right.$ and $\mathrm{Asn}^{245}$ Lys $\left.^{184}\right)$ and in a barrel-distal region of the POTRA2 domain $\left(\mathrm{Asp}^{165}\right.$-Lys $\left.{ }^{171}\right)$ were replaced with Ala or Cys, and the effects of these mutations on secretion activity were determined (Figure 2A-D). S-S bond formation is catalyzed by the periplasmic disulfide oxidase DsbA in the course of biogenesis, which generally affects SDSPAGE migration of the protein in the absence of a reducing agent, unless the intervening loop between the Cys residues is too short. The $\mathrm{Asn}^{245}$ Ala substitution markedly decreased the activity of FhaC and somewhat reduced its amount in the membrane, unlike formation of Cys ${ }^{157}-\mathrm{Cys}^{245}$ or $\mathrm{Cys}^{184}{ }^{18 y s^{245}} \mathrm{~S}-\mathrm{S}$ bonds (Figure 2C, D). This indicates that these barrel-POTRA2 interactions contribute to FhaC activity, possibly because they stabilize its conformation in the secretion cycle.

On the contrary, the engineered $\mathrm{Cys}^{165}+\mathrm{Cys}^{171}$ substitutions strongly reduced the level and the activity of FhaC in a $d s b A^{+}$background. Although protein migration was not affected, the S-S bond was most likely formed, since secretion was not reduced in a $d s b A^{-}$background or with the individual substitutions. The observation that $\mathrm{S}-\mathrm{S}$ bond formation between these two Cys residues is detrimental points to the need for flexibility in the barrel-distal region of the POTRA2 domain.

\section{Evidence for dynamics and alternative conformations of FhaC in lipid bilayers by NMR} spectroscopy 
To gain insight into the nature and the time scale of the conformational changes of FhaC, we made use of nuclear magnetic resonance (NMR) spectroscopy for its ability to characterize molecular structure and dynamics as well as minor conformational states of proteins in lipid bilayer environments (Mittermaier \& Kay, 2009; Liang \& Tamm, 2016). We recorded NMR spectra of FhaC in liposomes and lipid nanodiscs (Bayburt et al., 1998; Viegas et al., 2016), using solid- and solution-state NMR techniques, respectively. To render the $61-\mathrm{kDa}$ protein more accessible to NMR spectroscopy, we resorted to perdeuteration and specific ${ }^{1} \mathrm{H},{ }^{13} \mathrm{C}$-isotope labeling of isoleucine (Ile) $\delta_{1}$ methyl groups (Ruschak \& Kay, 2010). Since the 15 Ile residues of FhaC are well distributed across all structural elements of the protein (Figure 3A), we expected this reduced labeling scheme to nevertheless be able to report on larger-scale structural transitions of FhaC.

Signals from all Ile residues could be identified and assigned by Ile-to-Val mutations or paramagnetic relaxation enhancement experiments (Fig. 3B; see also below) (Amero et al., 2011; Venditti et al., 2011). The higher resolution of solution-state NMR spectra of FhaC in nanodiscs proved useful in the assignment (Figure 3 Supplement 1). Variable intensities of the Ile $\delta_{1}$ methyl signals report on local dynamics in the protein. While $\mathrm{Ile}^{14}$ in $\mathrm{H} 1$ was only visible in scalar coupling-based spectra, the signal of $\mathrm{Il}^{548}$ in $\beta$-strand B16 at the barrel seam consistently exhibited low intensity in both scalar and dipolar coupling-based spectra (Figure 3B, Figure 3 Supplement 1). This indicates sub- $\mu$ s time scale motion towards the N-terminus of the $\mathrm{H} 1$ helix and $\mu \mathrm{s}$-to-ms time scale exchange dynamics at the barrel seam, respectively. The notion of dynamics in FhaC is also supported by the absence of through-space correlations for all but the shortest Ile-Ile distances expected from the crystal structure (Figure 3 Supplement 2$)$. However, ${ }^{13} \mathrm{C}$ rotating-frame $\left(\mathrm{R}_{1 \rho}\right)$ relaxation dispersion experiments probing exchange between states with different chemical shifts on the $\mu$ s time scale (Lewandowski et al., 2011; Ma et al., 2014) yielded statistically flat dispersion 
142 profiles (Figure 3 Supplement 3), indicating that conformational changes of FhaC detectable by

143 Ile $\delta_{1}$ methyl chemical shifts must occur on slower time scales.

144 To specifically probe for alternative FhaC conformations, we performed paramagnetic 145 relaxation enhancement (PRE) NMR experiments in which a paramagnetic methanethiosulfonate 146 spin label (MTSL) is attached to an engineered Cys in the protein, and attenuation of NMR signals

147 of nuclei within a radius of about $25 \AA$ around the MTSL probe can be detected even if they only 148 transiently approach the probe (Battiste \& Wagner, 2000; Clore \& Iwahara, 2009; Nadaud et al., 149 2007). We chose residue 220 in the extracellular loop L1 for this experiment, yielding FhaC $220 \mathrm{R} 1$, 150 where R1 represents the spin label. Intensities of Ile $\delta_{1}$ methyl signals in FhaC ${ }^{220 R 1}$ measured by solid-state NMR in proteoliposomes were referenced to those in a sample with a diamagnetic

152 MTSL analog attached to the same residue, FhaC $220 \mathrm{R} 1$ dia $(F i g .3 \mathrm{~B}, \mathrm{C}$ ). Comparison of the signal intensity ratios obtained for different FhaC residues allows to determine whether a signal is more

154 attenuated than would be expected from the crystal structure, indicating a residue approaching the probe more closely (see Methods).

In agreement with expectations, residues more than $35 \AA$ away from the position of the 157 paramagnetic MTSL tag modelled onto the FhaC crystal structure $\left(\mathrm{Ile}^{114}\right.$, Ile ${ }^{136}$, and Ile ${ }^{141}$ in POTRA1 and POTRA2) exhibited the highest para- versus diamagnetic intensity ratios, while 159 residues expected to be within 16 to $25 \AA$ of the paramagnetic center (Ile ${ }^{27}, \mathrm{Ile}^{441}, \mathrm{Ile}^{506}, \mathrm{Ile}^{548}$ ) 160 showed attenuation of their NMR signals in paramagnetic FhaC ${ }^{220 R 1}$ (Figure 3B, C, Figure 3

161 Supplement 4). The overlapped signal corresponding to residues $\mathrm{Ile}^{252}$ and $\mathrm{Ile}^{420}$, at expected 162 distances to the paramagnetic center of 12 and $32 \AA$, respectively, exhibited intermediate 163 attenuation as expected. Signals from the POTRA2 H4 helix (Ile ${ }^{172}$, Ile ${ }^{176}$, Ile ${ }^{179}$ ) were not 164 significantly attenuated compared to reference signals. However, the signal of Ile ${ }^{188}$ in strand b5 
of the POTRA2 domain was attenuated more than would be expected for a residue at $35 \AA$ distance from the paramagnetic center. The difference in attenuation with respect to the reference residues $\mathrm{Ile}^{114}, \mathrm{Ile}^{136}$, and $\mathrm{Ile}^{141}$ is significant $(\mathrm{p}<0.05$, Figure $3 \mathrm{C})$. This result indicates that a region of the POTRA2 domain encompassing strand b5 can approach the extracellular loops of FhaC.

\section{Evidence for motions of the POTRA2 domain towards the extracellular side from EPR} spectroscopy

To complement the NMR data, we resorted to electron paramagnetic resonance (EPR) spectroscopy, another technique suitable to detect dynamics and minor conformational states of proteins in lipid bilayers, but sensitive to longer distances than can be measured by NMR (Sahu \& Lorigan, 2020; Torricella et al., 2021). Distances from about 1.8 to $8 \mathrm{~nm}$ between paramagnetic spin labels attached to membrane proteins can be measured with pulsed electron double resonance (PELDOR) EPR experiments and can provide insight into non-homogeneous conformational ensembles (Jeschke, 2012). Notably, in continuous-wave (CW) EPR spectroscopy experiments with FhaC carrying a single paramagnetic spin label attached at the solvent-exposed position 195 in the POTRA2 domain, we have previously observed a very slow-motion component for the spin probe (Guerin et al., 2015), suggesting possible interactions of the probe with the lipid bilayer in some conformers. To explore this further with explicit distance measurements, we performed PELDOR experiments. We introduced a Cys residue at position 503 in the extracellular L7 loop and combined it with another Cys either at position 195 in the b5-b6 hairpin of the POTRA2 domain, position 187 in the b5 strand, or position 33 in the linker, and we labeled both with an MTSL spin label, yielding FhaC variants FhaC ${ }^{33 R 1+503 R 1}$, FhaC ${ }^{187 R 1+503 R 1}$, and FhaC ${ }^{195 R 1+503 R 1}$. 
In b-octyl glucoside (bOG) micelles, for FhaC ${ }^{195 R 1+503 R 1}$ and $\mathrm{FhaC}^{187 \mathrm{R} 1+503 \mathrm{R} 1}$, the main populated states correspond to distance distributions between the two spin probes that are consistent with distances calculated using MTSL rotamer libraries attached to the corresponding residues of the FhaC crystal structure (Figure 4A; Figure 4 Supplement 1) (Jeschke, 2013, 2020). For FhaC $33 \mathrm{R} 1+503 \mathrm{R} 1$, a broad distance distribution was observed, with contributions centered at $4.2 \mathrm{~nm}$ and $4.6 \mathrm{~nm}$ as predicted by rotamer libraries (Figure 4 Supplement 1). In proteoliposomes, for FhaC ${ }^{195 R 1+503 R 1}$ and FhaC ${ }^{187 R 1+503 R 1}$, the main populated states correspond to long distances of 5 to $6 \mathrm{~nm}$ between the two spin probes (Figure 4B). Note that these distances are shorter than the expected distances calculated using MTSL rotamer libraries, since the lipid environment limited the dipolar evolution times that could be applied in PELDOR experiments (Figure 4 Supplement conformers with the two spin probes closer to one another than in the crystal structure conformation. Similarly, additional peaks corresponding to shorter-than-expected distances were observed in the distance distributions for $\mathrm{FhaC}^{187 \mathrm{R} 1+503 \mathrm{R} 1}$ (Figure 4B). These results strongly support the idea that the b5-b6 hairpin of the POTRA2 domain moves towards the extracellular surface of FhaC in some conformers. For FhaC ${ }^{33 R 1+503 R 1}$, distances both shorter and longer than

204 expected were obtained. This indicates that, in addition to moving away from the membrane when 205 H1 exits from the pore, as reported in (Guerin et al., 2014), the linker also moves toward the surface 206 in specific conformers.

207 The point mutation Asp ${ }^{492} \mathrm{Arg}$ disrupts a conserved salt bridge between L6 and the inner barrel wall and induces conformational changes in FhaC (Guerin et al., 2015). To determine whether it 209 affects the conformational equilibrium of the POTRA2 domain, we introduced the Asp ${ }^{492} \mathrm{Arg}$ 
substitution in FhaC ${ }^{195 R 1+503 R 1}$. Indeed, PELDOR experiments showed an increased proportion of species characterized by short inter-spin distances in this mutant (Figure 4 Supplement 3).

Notably, we did not obtain indications for alternative conformers in FhaC reconstituted into nanodiscs with EPR or NMR spectroscopy. Analysis of PELDOR experiments on FhaC ${ }^{195 R 1+503 R 1}$ in nanodiscs yielded only a long distance between the spin labels, as expected from the crystal structure, and PRE experiments on $\mathrm{FhaC}^{195 \mathrm{R} 1}$ in nanodiscs showed attenuation of NMR signals only within the POTRA2 domain (Figure 4 Supplement 4). Along with smaller linewidths of FhaC NMR signals in nanodiscs compared to liposomes (Figure 3 Supplement 1), these findings indicate that the constrained nanodisc environment limits the conformational space accessible to FhaC and hinders the larger-scale conformational changes that can be observed in proteoliposomes.

Taken together, both our EPR and NMR data show that the POTRA2 domain can undergo large conformational changes that bring its b5-b6 hairpin close to the membrane and the extracellular side, and that these conformational changes are facilitated by the rupture of the interaction between L6 and the inner barrel wall. The H1-POTRA1 linker can also adopt alternative conformations and notably move towards the cell surface.

\section{In vivo evidence for conformers with the POTRA2 domain or the linker close to surface loops}

To investigate whether the conformers observed in proteoliposomes also exist in vivo and to obtain insight into the potential position of the POTRA2 domain in those conformers, we simultaneously replaced two residues distant in the X-ray structure of FhaC with Cys residues to detect spontaneous S-S bond formation. Our rationale was that conformational changes that bring the two Cys residues close to each other should promote S-S bond formation even if the corresponding alternative conformations are short-lived, as the S-S bound species accumulate over time. These 
experiments were performed in a $d s b A^{-}$background, such that $\mathrm{S}-\mathrm{S}$ bonds formed after FhaC biogenesis and thus exclusively resulted from its conformational changes in the membrane. We combined Cys residues at the extracellular surface at positions 224 in L1, 290 in L3, 342 in L4, 391 in L5, 503 in L7 or 545 in L8 with periplasmic Cys residues in the POTRA2 domain at positions 167,176 or 195 , in the linker at position 48, or in the POTRA1 domain at position 86 (Figure 5A). None of the single Cys substitutions markedly affected the secretion activity of FhaC (Baud et al., 2014; Guerin et al., 2014; Guerin et al., 2015). Under non-reducing conditions, partial oxidation of FhaC as detected by aberrant migration in SDS-PAGE was identified for the combinations $\mathrm{Cys}^{195}+\mathrm{Cys}^{224}, \mathrm{Cys}^{176}+\mathrm{Cys}^{224}, \mathrm{Cys}^{48}+\mathrm{Cys}^{224}, \mathrm{Cys}^{48}+\mathrm{Cys}^{545}$ and weakly for $\mathrm{Cys}^{167}+\mathrm{Cys}^{224}$, indicating S-S bond formation within specific pairs of engineered Cys residues (Figure 5B). In contrast, no loop other than L1 or L8 was found to cross-link with those periplasmic regions, and none cross-linked with the Cys residue in the POTRA1 domain. To confirm S-S bond formation, the $\mathrm{FhaC}^{\mathrm{C} 48+\mathrm{C} 224}$ and $\mathrm{FhaC}^{\mathrm{C} 195+\mathrm{C} 224}$ variants were overexpressed, purified and subjected to liquid chromatography coupled to tandem mass spectrometry (MS) in reducing and nonreducing conditions. In both variant samples, the regions that contain the Cys residues were detected only when proteolytic digestion was performed after reduction and alkylation (Figure 5 Supplement 1), which supports S-S bond formation between the linker and L1 in FhaC ${ }^{\mathrm{C} 48+\mathrm{C} 224}$ and between the POTRA2 domain and $\mathrm{L} 1$ in $\mathrm{FhaC}^{\mathrm{C} 195+\mathrm{C} 224}$. Thus, in vivo, the last portion of the linker can be found close to the extracellular loops L1 and L8 that immediately follow and precede the first and last $\beta$-barrel strands, B1 and B16, respectively, and the $\alpha$ helix H4 and the b5-b6 $\beta$ hairpin of the POTRA2 domain can be found close to the extracellular loop L1. This indicates that these periplasmic elements approach the $\beta$-barrel seam in specific conformers, in agreement with our in vitro data. 
Interactions of portions of the POTRA2 domain with the $\beta$ barrel by native mass

\section{spectrometry}

259 Our data imply that the POTRA2 domain undergoes some breaking up in the secretion cycle. We in the gas phase can generally be related to the number of domains of a protein (Zhong et al., 2014), and extra-membrane domains are more likely to experience early unfolding than domains embedded in detergent or lipids due to collisional cooling (Barrera et al., 2009), those transitions might be caused by unfolding of the POTRA domains and/or ejection and unfolding of H1. Control

271 CIU experiments with other B. pertussis outer membrane proteins (OMPs) with small soluble

272 domains inside their $\beta$ barrels, the TonB-dependent transporter BfrG and the translocator domain 273 of an autotransporter, SphB1- $\alpha \beta$, showed a single unfolding transition at low voltage, which likely 274 corresponds to unfolding of these soluble domains (Figure 6 Supplement 2). Thus, the $\beta$ barrels of 275 these three proteins likely remain structurally intact at high activation conditions, most likely due to strong hydrogen bonding networks. 
which H1 is locked inside the barrel by an S-S bond and thus cannot move out (Guerin et al., 2014). FhaC ${ }^{\mathrm{C} 4+\mathrm{C} 391}$ exhibited the same transitions as wt FhaC, although the second unfolding event was delayed by $30 \mathrm{~V}$ and the overall CCS value was increased by $50 \AA^{2}$ (Figure 6 Supplement 3). As CIU is unlikely to break S-S bonds (Tian et al., 2015), comparison of these unfolding pathways suggests that the two transitions correspond to successive unfolding of the POTRA domains, with the barrel remaining intact in those conditions. H1 stays inside the barrel or its unfolding barely registers in the CCS values. The delay of the second unfolding transition for $\mathrm{FhaC}^{\mathrm{C} 4+\mathrm{C} 391}$ suggests that locking $\mathrm{H} 1$ in the barrel stabilizes one of the POTRA domains, although from the data we cannot discern which one.

We next tested the possibility that portions of the POTRA2 domain might bind to the $\beta$ barrel, probably along strands B1 or B16 upon opening of the barrel seam. Using native MS, we assessed the binding of synthetic peptides that correspond to various periplasmic portions of $\mathrm{FhaC}$, including b5-b6, b4+L (i.e., b4 followed by the b4-H3 linker) and $\mathrm{L}+\mathrm{H} 4$ (i.e., the $\mathrm{H} 3-\mathrm{H} 4$ linker followed by H4) of the POTRA2 domain, b2-b3 of the POTRA1 domain, Lk, a non-structured peptide from the linker region between $\mathrm{H} 1$ and the POTRA1 domain, and the $\mathrm{N}$-terminal $\beta$ hairpin of the FhaB transport substrate, Fha-NT (Figure 7; Figure 7 Supplement 1). The same experiments were performed with SphB1- $\alpha \beta$ to correct for non-specific binding, which might occur in native MS experiments due to artifacts induced by interaction with the detergent during the electrospray process (Landreh et al., 2016). Fha-NT, b4+L and b5-b6 exhibited binding to FhaC, with b5-b6 binding at the highest level and in two copies, but markedly less to the $\mathrm{FhaC}^{\mathrm{C} 4+\mathrm{C} 391}$ variant (Figure 7A; Figure 7 Supplement 2). In contrast, the peptides containing the sequences of b2-b3 of the POTRA1 domain, H4 or the H1-POTRA1 linker did not bind. 
We assessed structural changes induced by peptide binding using native ion-mobility (IM) MS. At low CE (i.e., no activation), all three peptides increased the CCS of the compact state of FhaC by rather small increments of $91-92 \AA^{2}$ (Figure 7B, C; Figure 7 Supplement 3 ). However, upon increasing the activation conditions, Fha-NT and b4+L no longer increased the CCS of FhaC, compared to the unbound protein. In contrast, the b5-b6 peptide caused an increase in CCS values both at low and high collisional activation, suggesting that a structural change was induced upon peptide binding and that the peptide was bound to a region that remains folded in these conditions unfold at high $\mathrm{CE}$, the effect of b5-b6 on the CCS might thus stem from peptide binding to the $\beta$ nevertheless caused a similar increase of CCS at both low and high energies, like with wt FhaC

312 (Figure 6 Supplement 3, Figure 7 Supplement 4). This supports the model that the peptide corresponding to the b5-b6 hairpin of the POTRA2 domain interacts with the $\beta$ barrel, and that this interaction is facilitated by the ejection of H1. Given the amphipathic nature of this hairpin, it most likely aligns with an edge of the open $\beta$-barrel seam, consistent with the cross-linking data.

\section{DISCUSSION}

318 As Omp85 transporters are thought to perform their functions in the absence of an energy source in the periplasm, their postulated conformational cycling must involve low energy barriers between conformers, as reported for BamA (Xiao et al., 2021). Here, we obtained evidence for large conformational changes of FhaC that involve portions of the POTRA2 domain approaching the extracellular side of the protein. Conformational changes of FhaC occur independently of the 
324 protein, with implications for its function. Notably, the conformational states appear to be in slow

325

326

327

328

329

330

331

332

333

334

335

336

337

338

339

340

341

342

343

344

345

346

equilibrium, as with BamA (Hartmann et al., 2018).

All structural elements of TpsB transporters are connected with one another, structurally and functionally, and their motions appear to be coupled (Guerin et al., 2014; Guerin et al., 2020; Guerin et al., 2015; Maier et al., 2015). In the resting conformation, H1 and L6 interact with the barrel wall, H1 with L1, the H1-POTRA1 linker with the POTRA domains, and the POTRA2 domain with the periplasmic side of the barrel (Guerin et al., 2020; Maier et al., 2015). In the secretion process, L6 breaks its connection with the barrel wall, H1 moves towards the periplasm, and part of the B1-B16 seam unzips (Guerin et al., 2014; Guerin et al., 2020; Guerin et al., 2015). In the current work we have obtained evidence fur further conformational changes involving the POTRA2 domain. Thus, EPR, NMR and S-S cross-linking data revealed the proximity of parts of the POTRA2 domain to the barrel seam and to the extracellular side of FhaC in specific conformers, and structural MS experiments showed the binding of the POTRA2 b5-b6 hairpin peptide to the $\beta$ barrel under conditions in which the POTRA domains are very likely unfolded.

Based on these and previous data, we propose the following model for the first steps of secretion (Figure 8). The closed, resting conformation of $\mathrm{FhaC}$ is in slow equilibrium with an open conformation in which L6 has been released from its interaction with the barrel wall and H1 has moved out of the pore (Figure 8A) (Guerin et al., 2014; Guerin et al., 2020; Guerin et al., 2015). In the open conformation, the linker has vacated the substrate binding site on the POTRA2 domain, thus enabling a specific portion of the conserved TPS domain of the substrate to bind to the groove between H4 and b5 (Delattre et al., 2011). According to molecular dynamics simulations, the exit of $\mathrm{H} 1$ facilitates barrel unzipping between B1 and B16 (Guerin et al., 2020). Barrel unzipping is coupled with a swing motion of the b5-b6 hairpin of the POTRA2 domain towards the barrel, 
347 followed by its binding to the open seam, probably by $\beta$ augmentation, thus enlarging the barrel

348 and hoisting the bound substrate into the channel (Figure 8B).

349 The next secretion steps are more speculative. The entropic cost of confining a portion of the 350 bound, unfolded polypeptide inside the channel might facilitate its diffusion toward the surface 351 (Halladin et al., 2021). The TPS domain most likely forms a hairpin inside the barrel, as proposed 352 previously (Nash \& Cotter, 2019), and a specific region binds to the extracellular $\beta$ sheet formed by B5-B8 (Baud et al., 2014), which might template the folding of the substrate into a nascent $\beta$ helix. The substrate would progressively be threaded across the channel and fold at the surface, leading to the formation of a stable $\beta$ helix nucleus (Alsteens et al., 2013), which would prevent backtracking of the protein chain, effectively adding directionality to its stochastic motion as proposed by the "Brownian ratchet" mechanism (Peterson et al., 2010). According to our model, TpsB transporters mediate protein secretion without the need for cofactors by utilizing intrinsic 359 protein dynamics. Although the conformational changes we describe are unprecedented in the Omp85 superfamily, divergent functional evolution has necessarily led to specific mechanistic adaptations. Recent work has indicated that energy may be transduced from the inner membrane to the BAM complex through the protonmotive force-utilizing SecDF complex (Alvira et al., 2020). One cannot rule out that the intrinsic conformational changes of FhaC in vivo are similarly enhanced by an energy-transducing mechanism.

The b5-b6 hairpin most likely binds by $\beta$ augmentation of the barrel strand B1, by analogy with BamA and Sam50 in which the unzipped B1 strand templates folding of client proteins by $\beta$ 367 augmentation (Doyle \& Bernstein, 2019; Höhr et al., 2018; Tomasek et al., 2020; Wu et al., 2021). 368 This mode of binding is supported by our CIU results showing an increased cross-section of the 369 protein upon binding of b5-b6 to the barrel and by the in vivo formation of an S-S bond between 
370 the tip of that hairpin and the extracellular loop L1. The b5-b6 sequence, with its amphipathic

371 nature and suitable charge partitioning, fits ideally in the open seam. The observation that portions

372 of the linker may approach the extracellular loops likely reflects futile conformational changes

373 involving the linker bound to the POTRA2 being hoisted into the channel in place of the substrate,

374 in the absence of the latter (Figure 8C). Notably, evidence for the C-terminal portion of the linker

375 reaching the cell surface was obtained previously (Guedin et al., 2000).

376 As the POTRA2 domain partially breaks up during secretion, it must reassemble after secretion

377 is completed. This may be mediated by the interactions of the H3 helix and the barrel-proximal

378 end of $\mathrm{b} 5$ of the POTRA2 domain with the periplasmic turn T1 of the barrel, which are important

379 for FhaC activity. These fixed points of the POTRA2 domain may ensure that FhaC can regain its

380 resting conformation after secretion, which is necessary to limit outer membrane permeability.

381 Consistent with this hypothesis, disrupting the conformation of the periplasmic turn T1 yielded

382 transient, very large channels as detected in electrophysiology experiments (Méli et al., 2006).

383 Stabilizing specific conformations of the transporter might also account for the importance of the

384 interaction between L6 and the inner barrel wall (Delattre et al., 2010).

385 In summary, we propose a novel mechanism of protein transport in TPS systems based on

386 large-scale, spontaneous conformational dynamics of the TpsB partner. Our model integrates and

387 explains currently available data from several complementary in vitro and in vivo approaches and

388 establishes mechanistic links between TpsBs and other Omp85 transporters. 
E. coli JCB570 or JCB571 (dsbA::kan) were used for low level expression of FhaC and E. coli

BL21(DE3-omp5) for overexpression. For peptide mapping FhaC ${ }^{\mathrm{C} 48+\mathrm{C} 224}$ and $\mathrm{FhaC}^{\mathrm{C} 195+\mathrm{C} 224}$ were

overexpressed in BL21(DE3-omp5 dsbA::kan), which was constructed as described in (Derbise et performed from pET22 or pET24 plasmids (Clantin et al., 2007). ptacFha44-His codes for the first adding a 1.2-kb Sal-BamHI fragment of the $f h a B$ gene into the same sites of ptacNM2lk-His

(Guerin et al., 2015). pFJD63 codes for FhaC under the control of the $\mathrm{P}_{\mathrm{BAD}}$ promoter (Guedin et of pFJD63 with the XbaI-HindIII fhaC fragments carrying the relevant mutations from the pFc3

pSphB $1 \alpha \beta$ is a derivative of $\mathrm{pT7SB} \alpha \beta$ (Dé et al., 2008) with a 6-His tag. To construct pT7bfrG-

(Méli et al., 2006) after the signal-peptide and 6-His tag sequences.

\section{In vivo assays}

409 To monitor S-S bond formation in vivo, the $\mathrm{pFc} 3$ variants were introduced in E. coli JCB571. The

410 recombinant bacteria were grown at $37^{\circ} \mathrm{C}$ in minimum $\mathrm{M} 9$ medium containing $0.1 \%$

411 casaminoacids under agitation. The cells were collected by centrifugation when the optical

412 densities at $600 \mathrm{~nm}\left(\mathrm{OD}_{600}\right)$ of the cultures reached 0.8 . The cell pellets were resuspended in 50

$413 \mathrm{mM}$ sodium $(\mathrm{pH}$ 6.8) containing $10 \mathrm{mM} \mathrm{N}$-ethylmaleimide and lysed using a Hybaid ribolyzer 414 apparatus (50 sec at speed 6). The membranes were collected by ultracentrifugation of the clarified 
415 lysates at 90,000 g for $1 \mathrm{~h}$. The pellets were resuspended in loading buffer without reducing agent 416 and separated into two aliquots, with DTE added at $25 \mathrm{mM}$ to one of them before heating at $70^{\circ} \mathrm{C}$

417 for $10 \mathrm{~min}$. FhaC was detected using anti-FhaC antibodies (Delattre et al., 2011) with alkaline 418 phosphatase development for $15 \mathrm{~min}$.

419 For the secretion assays, overnight cultures of E. coli JCB570 or JCB571 harboring a pFJD63

420 derivative and ptacFha44-His were diluted to $\mathrm{OD}_{600}$ of 0.3 in LB and grown under agitation with $4210.01 \%$ arabinose for $20 \mathrm{~min}$ to produce FhaC. The bacteria were collected by centrifugation, 422 resuspended in prewarmed LB without arabinose and grown to OD 600 of 0.8 before adding IPTG 423 at $1 \mathrm{mM}$ to induce the expression of Fha44. Culture aliquots were collected 5 and 20 min thereafter 424 and placed on ice. After centrifugation to harvest the bacteria, Fha44 was affinity-purified from 425 the supernatants with Ni-NTA beads (Qiagen, Courtaboeuf, France). The membrane extracts were 426 prepared and FhaC was detected as above. Fha44 was detected by immunoblotting using anti-6His 427 antibodies, the ECL kit of Amersham (Merck, St Quentin-Fallavier, France) and the Amersham 428 Imager 600 (GE) with $1 \mathrm{sec}$ exposure. The amounts of Fha44 in supernatants were quantified with 429 ImageJ.

\section{Protein Purification and spin labeling}

432 The production and purification of the FhaC derivatives was performed as described (Guerin et al., 433 2014). Expression for NMR experiments was performed in $\mathrm{M} 9$ minimal medium in $\mathrm{D}_{2} \mathrm{O}, 2.5 \mathrm{~g} / \mathrm{L}$

$434{ }^{2} \mathrm{H}$-glucose (Sigma, St Quentin-Fallaviers, France), $1 \mathrm{~g} / \mathrm{L}{ }^{15} \mathrm{~N}-\mathrm{NH} 4 \mathrm{Cl}, 1 \mathrm{~g} / \mathrm{L}{ }^{15} \mathrm{~N},{ }^{2} \mathrm{H}$-isogro (Sigma) 435 and ${ }^{13} \mathrm{C}-\alpha$-ketobutyric acid (Sigma) to achieve $\mathrm{u}-\left({ }^{2} \mathrm{H},{ }^{15} \mathrm{~N}\right)$, Ile- $\delta_{1}\left({ }^{13} \mathrm{CH}_{3}\right)$ isotope labeling (Ruschak $436 \&$ Kay, 2010). For spin labeling, $3 \mathrm{mM}$ tris(2-carboxyethyl)phosphine (TCEP, Sigma) was added 437 to the detergent extract before ion exchange chromatography. The FhaC-containing fractions were 
438 mixed with a 10-fold molar excess (1-oxyl-2,2,5,5-tetramethyl- $\Delta 3$-pyrroline-3-methyl) methanethiosulfonate (MTSL) or its diamagnetic analogue (1-Acetoxy-2,2,5,5-tetramethyl- $\delta$-3pyrroline-3-methyl) methanethiosulfonate (Toronto Research Chemicals, North York, ON,

441 Canada) at $15^{\circ} \mathrm{C}$ with gentle agitation for 16 hours. Excess MTSL was removed by

442 chromatography. SphB1- $\alpha \beta$ and BfrG were produced from E. coli BL21(DE3-omp5) and purified

443 from bOG extracts using $\mathrm{Ni}^{2+}$ affinity chromatography. For BfrG $300 \mathrm{mM} \mathrm{NaCl}$ was added to

444 improve solubility.

445

\section{Preparation of liposomes and nanodiscs and protein reconstitution}

447 Small unilamellar vesicles (SUVs) of E. coli polar lipids were prepared as described (Guerin et al., 2014). The SUVs were mixed with FhaC variants at lipid:protein molar ratios of approx. 2500:1

449 for EPR and 200:1 for NMR experiments, respectively, at room temperature, with gentle agitation

450 for one hour. The proteoliposomes were formed by removal of detergent with the progressive

451 addition of Biobeads SM2 (Bio-Rad), and the proteoliposomes were collected by 452 ultracentrifugation. All steps were performed under argon. Final buffer concentrations after mixing

453 FhaC and liposomes were about $12.5 \mathrm{mM}$ each of Tris- $\mathrm{HCl}$ and $\mathrm{NaP}_{\mathrm{i}}, 150 \mathrm{mM} \mathrm{NaCl}, \mathrm{pH}$ 6.7.

$454 \quad$ Nanodiscs were prepared with the MSP1D1 and MSP1E3D1 scaffold proteins (Ritchie et al., $4552009)$ produced in E. coli BL21(DE3), with an induction of $3 \mathrm{~h}$ at $28^{\circ} \mathrm{C}$. For NMR experiments, 456 scaffold proteins were expressed in $\mathrm{M} 9$ minimal medium in $\mathrm{D}_{2} \mathrm{O}$ using ${ }^{2} \mathrm{H}$-glucose as carbon 457 source to suppress their signals in the $\left({ }^{1} \mathrm{H},{ }^{13} \mathrm{C}\right)$-based NMR spectra. The bacteria were broken using 458 a French press in $50 \mathrm{mM}$ Tris- $\mathrm{HCl}(\mathrm{pH}$ 8), $300 \mathrm{mM} \mathrm{NaCl}$ (TN buffer), 1\% Triton X100 (TNX

459 buffer), and the clarified lysates were subjected to $\mathrm{Ni}^{2+}$ affinity chromatography. After successive 
461

462

463

464

465

466

467

468

469

470

471

472

\section{$473 \quad$ NMR experiments}

474 For solid-state NMR experiments on FhaC variants reconstituted into liposomes, the

475 proteoliposomes collected by ultracentrifugation were transferred to $1.3 \mathrm{~mm}$ magic-angle-spinning

476 (MAS) solid-state NMR rotors (Bruker Biospin, Wissembourg, France) using an

477 ultracentrifugation device (Bertini et al., 2012) (Giotto Biotech, Sesto Fiorentino, Italy) in a

478 Beckman ultracentrifuge (SW 32 Ti rotor, $77,000 \times \mathrm{g}, 12^{\circ} \mathrm{C}, 30-60 \mathrm{~min}$ ). NMR experiments were

479 performed on spectrometers operating at 800 and $950 \mathrm{MHz}{ }^{1} \mathrm{H}$ Larmor frequency (18.8 and 22.3

480 T magnetic field) (Bruker Biospin) at a MAS frequency of $50 \mathrm{kHz}$. Sample temperature was kept

481 at about $17^{\circ} \mathrm{C}$ as judged by the chemical shift of the bulk water resonance. Spectra were indirectly

482 referenced to 2,2-dimethyl-2-silapentane-5-sulfonate (DSS) via the lipid methylene proton

resonance, which appears at $1.225 \mathrm{ppm}$ under our experimental conditions. Typical pulse lengths 
484 for ${ }^{1} \mathrm{H}$ and ${ }^{13} \mathrm{C}$ hard $90^{\circ}$ pulses were 2.1 and $3.8 \mu$ s, respectively. For cross-polarization (CP), field

485

486

487

488

489

490

491

492

493

494

495

496

497

498

499

500

501

502

503

504

505

506 strengths were 21 and $30 \mathrm{kHz}$ for ${ }^{1} \mathrm{H}$ and ${ }^{13} \mathrm{C}$, respectively $(\mathrm{n}=1$ double-quantum Hartmann-Hahn condition), with a 50-to-100\% ramp on the ${ }^{1} \mathrm{H}$ radiofrequency (RF) field and a duration of $1.5 \mathrm{~ms}$.

${ }^{1} \mathrm{H}$-detected 2D ${ }^{13} \mathrm{C}-{ }^{1} \mathrm{H}$ dipolar hCH correlation spectra (Barbet-Massin et al., 2014) were typically

recorded with 1600 data points and a spectral width of $40 \mathrm{ppm}$ in the direct ${ }^{1} \mathrm{H}$ dimension and 100 to 140 data points and a spectral width of $13 \mathrm{ppm}$ in the indirect ${ }^{13} \mathrm{C}$ dimension. For water suppression, the MISSISSIPPI scheme (Zhou \& Rienstra, 2008) at $15 \mathrm{kHz}{ }^{1} \mathrm{H}$ RF field with a duration of typically $200 \mathrm{~ms}$ was employed. For the $2 \mathrm{D}$ hChH correlation spectrum, a ${ }^{1} \mathrm{H}-{ }^{1} \mathrm{H}$ mixing time of $6.4 \mathrm{~ms}$ using radio frequency driven recoupling (Bennett et al., 1992) with a ${ }^{1} \mathrm{H}$ field strength of $120 \mathrm{kHz}$ was applied between back-CP and acquisition. ${ }^{13} \mathrm{C} \mathrm{R}_{1 \rho}$ spectra (Lewandowski et al., 2011; Ma et al., 2014) were recorded in a pseudo-3D fashion, with the ${ }^{13} \mathrm{C}$ spinlock period inserted between the initial $\mathrm{CP}$ and the ${ }^{13} \mathrm{C}$ indirect evolution of the hCH sequence. Spinlock field strengths from 1.2 to $10 \mathrm{kHz}$ were used, and 5 spinlock durations from 2.5 to $80 \mathrm{~ms}$ with one repeated value were recorded for each spinlock. The spinlock carrier frequency was kept at the center of the isoleucine $\delta_{1}$ methyl ${ }^{13} \mathrm{C}$ region, as in all other hCH correlation spectra. A ${ }^{1} \mathrm{H}$ $180^{\circ}$ pulse was inserted in the middle of the spinlock period to suppress chemical shift anisotropy / dipolar coupling cross-correlated relaxation (Kurauskas et al., 2016). Solid-state PRE NMR experiments were recorded on FhaC samples with either a paramagnetic MTSL tag or a diamagnetic MTSL analogue (Nadaud et al., 2007) attached to a Cys, reconstituted into E. coli polar lipid liposomes. Standard dipolar 2D hCH correlation spectra were recorded.

Solution-state NMR experiments on FhaC in nanodiscs were conducted on a $900 \mathrm{MHz}$ spectrometer (Bruker Biospin) at $32^{\circ} \mathrm{C}$ sample temperature. Standard ${ }^{13} \mathrm{C}-{ }^{1} \mathrm{H}$ heteronuclear multiple-quantum coherence (HMQC) or SOFAST-HMQC (Schanda \& Brutscher, 2005) 
507

508

509

510

511

512

513

514

515

516

517

518

519

520

521

522

523

524

525

526

527

528

529

experiments were recorded with 2048 and 150 data points and spectral widths of 14 and 7.4 ppm in direct ${ }^{1} \mathrm{H}$ and indirect ${ }^{13} \mathrm{C}$ dimensions, respectively. For PRE experiments, standard ${ }^{13} \mathrm{C}-{ }^{1} \mathrm{H}$ HMQC spectra were recorded on a FhaC ${ }^{195 R 1}$ sample before and after reduction of the paramagnetic MTSL tag with a 10-fold molar excess of ascorbic acid (Battiste \& Wagner, 2000).

NMR spectra were processed with TopSpin 4.0.3 (Bruker Biospin) or NMRPipe (Delaglio et al., 1995) and analyzed with Sparky (Lee et al., 2015) or CcpNMR (Vranken et al., 2005). For the relaxation dispersion curves, effective transverse relaxation rates $R_{2, \text { eff }}$ (comprising intrinsic transverse relaxation rate $R_{2,0}$ and any exchange contribution $R_{\mathrm{ex}}$ ) were extracted from experimental $R_{1 \rho}$ values using separately recorded $R_{1}$ experiments (Palmer \& Massi, 2006). For PRE experiments, ratios of peak intensities in spectra of para- and diamagnetic species (FhaC ${ }^{220 R 1}$ and $\mathrm{FhaC}^{220 \mathrm{R} 1 \mathrm{dia}}$ for solid-state experiments, oxidized and reduced $\mathrm{FhaC}^{195 \mathrm{R} 1}$ in case of the solution-state experiments, respectively) were calculated. These para- versus diamagnetic signal intensity ratios do not normalize to 1 in our case. In the solid-state experiments, this is most likely due to variations between the samples in terms of efficiency of protein reconstitution into liposomes and total amounts of sample transferred to the NMR rotor. Both in solid and solution state, spectroscopic factors likely also play a role (incomplete longitudinal relaxation and thus lower signal-to-noise in the spectra of diamagnetic samples due to the use of short inter-scan delays of $1 \mathrm{~s}$ (Iwahara et al., 2007)). We have thus opted to normalize PRE ratios to the maximum ratio observed in each experiment, which was always observed in one of the residues furthest from the paramagnetic center $\left(\mathrm{Ile}^{136}\right.$ in $\mathrm{FhaC}^{220 \mathrm{R} 1}$, $\mathrm{Ile}^{14}$ in $\left.\mathrm{FhaC}^{195 \mathrm{R} 1}\right)$. This is equivalent to normalizing signals within each spectrum to a reference signal whose intensity is unaffected by PRE effects. We then only analyzed relative signal attenuation levels, instead of attempting to extract quantitative distance measures. Error bars of PRE intensity ratios were calculated based on spectral 
530

531

532

533

534

535

536

537

538

539

540

541

542

543

544

545

546

547

548

549

550

551

552

noise levels (root-mean-standard deviation of the spectral noise) using standard error propagation.

To estimate expected distances between FhaC Ile residues and the MTSL label on residue 220, and consequently relative PRE attenuation levels, an ensemble of 200 MTSL conformations compatible with labeling on FhaC residue 220 was calculated using the mtsslSuite web server (Hagelueken et al., 2015; Hagelueken et al., 2012) (http://www.mtsslsuite.isb.ukbonn.de/) and the FhaC crystal structure (PDB 4QKY) with residue 220 changed to Cys in PyMOL (The PyMOL Molecular Graphics System. Schrödinger, LLC). The average position of the paramagnetic center (taken as halfway between nitrogen and oxygen atoms of the MTSL nitroxide ring) was calculated from the coordinates of these 200 conformations; distances from that position to Ile $\mathrm{C} \delta 1$ nuclei were calculated using PyMOL.

\section{EPR Experiments}

PELDOR experiments were performed at Q-band frequency ( $34 \mathrm{GHz})$ using a Bruker EleXsys E580 spectrometer equipped with an overcoupled Bruker EN 5107D2 resonator. Pulses were generated with a Bruker SpinJet AWG and amplified with a $50 \mathrm{~W}$ TWT amplifier. The experiments were performed at $50 \mathrm{~K}$ and $30 \mathrm{~K}$ using a variable-temperature cryogen-free system (Oxford, Oxford, UK). The deadtime-free, four-pulse PELDOR sequence $[(\pi / 2)$ probe $-\tau 1-$ $(\pi)$ probe $-\tau 1+\mathrm{t}-(\pi)$ pump $-\tau 2-\mathrm{t}-(\pi)$ probe $-\tau 2-($ echo $)]$ was employed with a 200-ns $\tau 1$ delay and $\tau 2$ delays ranging from 3,200 ns to 7,000 ns depending on the sample (Pannier et al., 2000). Probe pulses were $10 \mathrm{~ns}(\pi / 2)$ and $20 \mathrm{~ns}(\pi)$ Gaussian-shaped pulses at a frequency corresponding to the maximum of the resonator response function and a magnetic field value corresponding to the high-field shoulder of the echo-detected field-swept spectrum. The pump pulse was implemented as a 24-ns pulse centered at a frequency $55 \mathrm{MHz}$ higher than the probe 
553 frequency and corresponding to the maximum of the nitroxide field-swept spectrum. Raw time-

554 domain PELDOR traces were background-corrected using the DeerAnalysis 2019 package

555 (Jeschke et al., 2006), and the resulting signals were power-scaled in MATLAB to suppress sum

556 and difference peaks arising from multispin effects. Distance distributions were then calculated

557 from the scaled and background-corrected PELDOR traces by Tikhonov regularization. For

$558 \mathrm{FhaC}^{33 \mathrm{R} 1+503 \mathrm{R} 1}$, FhaC ${ }^{187 \mathrm{R} 1+503 \mathrm{R} 1}$ and $\mathrm{FhaC}^{195 \mathrm{R} 1+503 \mathrm{R} 1}$, distance distributions were predicted using a

559 pre-computed rotamer library of the MTSL spin probe attached to specific residues on the PDB

560 structure (Jeschke, 2020).

561

\section{Mass fingerprinting of FhaC variants}

563 Purified FhaC ${ }^{\mathrm{C} 48+\mathrm{C} 224}$ and $\mathrm{FhaC}^{\mathrm{C} 195+\mathrm{C} 224}$ variants were subjected to non-reducing SDS-PAGE, and 564 acrylamide bands corresponding to the oxidized forms of the two proteins were excised. They were 565 washed with $50 \mu \mathrm{L}$ of acetonitrile $(\mathrm{ACN}) / \mathrm{NH}_{4} \mathrm{HCO}_{3}(75 / 25)$ four times and dehydrated with $\mathrm{ACN}$, 566 or incubated in $10 \mathrm{mM} \mathrm{DTT}$ in $\mathrm{NH}_{4} \mathrm{HCO}_{3}$ for $30 \mathrm{~min}$ at $57^{\circ} \mathrm{C}$ and $30 \mathrm{~min}$ room temperature, 567 followed by incubation in $55 \mathrm{mM}$ iodoacetamide in $25 \mathrm{mM} \mathrm{NH}_{4} \mathrm{HCO}_{3}$ for 20 min in the dark, 3 568 washes with $\mathrm{NH}_{4} \mathrm{HCO}_{3}$ and dehydration with $\mathrm{ACN}$ performed twice. The $\mathrm{pH}$ of the samples was 569 decreased to 2.0, digestion was performed with pepsin $(0.01 \mu \mathrm{g} / \mu \mathrm{L})$ (Promega, Charbonnieres-les570 Bains, France) at a 1:50 enzyme:substrate ratio at $37^{\circ} \mathrm{C}$ for 3 hours, and the reaction was stopped 571 by heating at $95^{\circ} \mathrm{C}$ for $10 \mathrm{~min}$.

572 NanoLC-MS/MS analysis was performed using a nanoAcquity Ultra-Performance-LC (Waters, 573 Manchester, UK) coupled to a Q-Exactive Plus Orbitrap mass spectrometer (Thermo Scientific, 574 Illkirch, France). Peptides were trapped on a nanoACQUITY UPLC precolumn (C18, $180 \mu \mathrm{m} x$ $57520 \mathrm{~mm}, 5 \mu \mathrm{m}$ particle size), and eluted from a nanoACQUITY UPLC column (C18, $75 \mu \mathrm{m} \times 250$ 
$\mathrm{mm}, 1.7 \mu \mathrm{m}$ particle size) at a constant temperature of $60^{\circ} \mathrm{C}$. Mobile phases $\mathrm{A}$ and $\mathrm{B}$ were composed of $0.1 \%$ formic acid in water and $0.1 \%$ formic acid in $\mathrm{ACN}$, respectively. Peptides were eluted with gradients of B from 1 to $8 \%$ for $2 \mathrm{~min}, 8$ to $35 \%$ for $58 \mathrm{~min}, 35$ to $90 \%$ for $1 \mathrm{~min}, 90 \%$ for $5 \mathrm{~min}, 90$ to $1 \% \mathrm{~B}$ for $1 \mathrm{~min}$ and a concentration of $1 \% \mathrm{~B}$ for $20 \mathrm{~min}$, with a constant flow rate of $400 \mathrm{~nL} / \mathrm{min}$. The source temperature of the mass spectrometer was set to $250^{\circ} \mathrm{C}$ and the spray voltage at $1.8 \mathrm{kV}$. Full scan MS spectra were acquired in positive mode with a resolution of 140,000, a maximum injection time of $50 \mathrm{~ms}$, and an AGC target value of $3 \times 10^{6}$ charges. The 10 most intense multiply charged peptides per full scan were isolated using a $2 \mathrm{~m} / \mathrm{z}$ window and fragmented using higher energy collisional dissociation (normalized collision energy of 27). MS/MS spectra were acquired with a resolution of 17,500, a maximum injection time of $100 \mathrm{~ms}$ and an AGC target value of $1 \times 10^{5}$, and dynamic exclusion was set to $60 \mathrm{sec}$. The system was fully controlled by XCalibur software v3.0.63, 2013 (Thermo Scientific) and NanoAcquity UPLC console v1.51.3347 (Waters). The MS/MS data were interpreted using a local Mascot server with MASCOT 2.5.0 algorithm (Matrix Science, London, UK). Spectra were searched with a mass tolerance of $5 \mathrm{ppm}$ for MS and 0.07 Da for MS/MS data, using none as enzyme. Oxidation $(+15.99$ $\mathrm{Da})$, and carbamidomethylation $(57.02 \mathrm{Da})$ were specified as variable modifications. Protein identifications were validated with a Mascot ion score above 25.

\section{Native MS and ion mobility}

Purified FhaC was buffer exchanged into $100 \mathrm{mM}$ ammonium acetate buffer, $\mathrm{pH}$ 6.8, supplemented with $50 \mathrm{mM}$ bOG using a P6 desalting column (Biorad, Marnes-la-Coquette, France). Samples were directly infused with nano-electrospray ionization with in-house-prepared gold-coated borosilicate glass capillaries with a spray voltage of $+1.4 \mathrm{kV}$. Spectra were recorded 

binding.

on a quadrupole TOF instrument (Synapt G2 HDMS with 32K quadrupole, Waters) optimized for transmission of native, high-m/z protein assemblies. Critical voltages and pressures throughout the instrument were $50 \mathrm{~V}, 10 \mathrm{~V}, 150 \mathrm{~V}$ and $15 \mathrm{~V}$ for the sampling cone, extraction cone, trap and transfer collision cell, respectively, with pressures of 9 mbar, $1.47 \times 10^{-2}$ mbar and $1.21 \times 10^{-2}$ mbar for the source, trap and transfer regions unless indicated otherwise. CIU ion mobility experiments were performed with $50 \mathrm{~V}$ sampling cone; 50-200 V trap collision energy; $42 \mathrm{~V}$ trap DC bias; and $15 \mathrm{~V}$ transfer collision energy. Pressures throughout the instrument were 9 and 1.46 $\times 10^{-2} \mathrm{mbar}$ for the source and trap/transfer collision cells. All spectra were processed with Masslynx v4.1 (Waters). Collision cross section calibration was performed using GDH, ADH, ConA and PK as proteins standard as described (Allen et al., 2016). It should be noted that due to the generally lower charge states observed for membrane proteins, and the increased collision energies required (compared to soluble proteins) for gentle release of proteins from detergent micelles, the CCS values reported here are less accurate and intended for qualitative comparison rather than quantitative matching to theoretical models.

\section{Peptide binding assays}

Synthesized peptides were dissolved in DMSO to a final concentration of $100 \mathrm{mM}$ and added to the protein sample at final concentrations of $10 \mu \mathrm{M}$ FhaC and $100 \mu \mathrm{M}$ peptide. To correct for non-

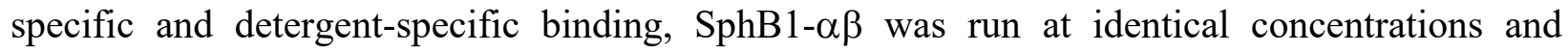
conditions. For both proteins the fraction of peptide-bound protein was calculated based on peak intensities, after which the binding to the decoy protein was subtracted to correct for non-specific 


\section{Acknowledgements}

We thank Xavier Hanoulle for useful suggestions and discussions at the start of this project and Isabelle Landrieu for her support and advice. This work was funded by the Agence Nationale de Recherche grant ANR-17-CE11-0043-02 "OPEN_BAR" to FJD and a "Projets exploratoires premier soutien (PEPS)" grant by the CNRS and the University of Lille to RS. Financial support and spectrometer access by the IR-RMN-THC FR 3050 CNRS for conducting NMR experiments at the IR-RMN platforms in Lille and Grenoble is gratefully acknowledged. EPR experiments were performed within the national facility RENARD at the University of Lille (Federation IR-RPE 3443). Support by the Centre National de la Recherche Scientifique (CNRS), Université de Strasbourg (Unistra) and the French Proteomic Infrastructure (ProFI; ANR-10-INBS-08-03) is acknowledged. The authors would also like to thank the IdeX program of the University of Strasbourg for funding the Synapt G2Si instrument.

\section{References}

Allen, S. J., Eaton, R. M., \& Bush, M. F. (2016). Analysis of native-like ions using structures for lossless ion manipulations. Anal Chem, 88(18), 9118-9126. https://doi.org/10.1021/acs.analchem.6b02089

Alsteens, D., Martinez, N., Jamin, M., \& Jacob-Dubuisson, F. (2013). Sequential unfolding of Beta helical protein by single-molecule atomic force microscopy. PLOS ONE, 8(8), e73572. https://doi.org/10.1371/journal.pone.0073572

PONE-D-12-39879 [pii]

Alvira, S., Watkins, D. W., Troman, L., Allen, W. J., Lorriman, J. S., Degliesposti, G., Cohen, E. J., Beeby, M., Daum, B., Gold, V. A., Skehel, J. M., \& Collinson, I. (2020). Inter-membrane association of the Sec and BAM translocons for bacterial outer-membrane biogenesis. Elife, 9. https://doi.org/10.7554/eLife.60669

Amero, C., Asuncion Dura, M., Noirclerc-Savoye, M., Perollier, A., Gallet, B., Plevin, M. J., Vernet, T., Franzetti, B., \& Boisbouvier, J. (2011). A systematic mutagenesis-driven strategy for site-resolved NMR studies of supramolecular assemblies. I Biomol NMR, 50(3), 229-236. https://doi.org/10.1007/s10858-011-9513-5 
665

666

667

668

669

670

671

672

673

674

675

676

677

678

679

680

681

682

683

684

685

686

687

688

689

690

691

692

693

694

Barbet-Massin, E., Pell, A. J., Retel, J. S., Andreas, L. B., Jaudzems, K., Franks, W. T., Nieuwkoop, A. J., Hiller, M., Higman, V., Guerry, P., Bertarello, A., Knight, M. J., Felletti, M., Le Marchand, T., Kotelovica, S., Akopjana, I., Tars, K., Stoppini, M., Bellotti, V., Bolognesi, M., Ricagno, S., Chou, J. J., Griffin, R. G., Oschkinat, H., Lesage, A., Emsley, L., Herrmann, T., \& Pintacuda, G. (2014). Rapid protondetected NMR assignment for proteins with fast magic angle spinning. J Am Chem Soc, 136(35), 12489-12497. https://doi.org/10.1021/ja507382j

Barrera, N. P., Isaacson, S. C., Zhou, M., Bavro, V. N., Welch, A., Schaedler, T. A., Seeger, M. A., Miguel, R. N., Korkhov, V. M., van Veen, H. W., Venter, H., Walmsley, A. R., Tate, C. G., \& Robinson, C. V. (2009). Mass spectrometry of membrane transporters reveals subunit stoichiometry and interactions. Nat Methods, 6(8), 585-587. https://doi.org/10.1038/nmeth.1347

Battiste, J. L., \& Wagner, G. (2000). Utilization of site-directed spin labeling and high-resolution heteronuclear nuclear magnetic resonance for global fold determination of large proteins with limited nuclear overhauser effect data. Biochemistry, 39(18), 5355-5365. https://doi.org/10.1021/bi000060h

Baud, C., Guerin, J., Petit, E., Lesne, E., Dupre, E., Locht, C., \& Jacob-Dubuisson, F. (2014). Translocation path of a substrate protein through its Omp85 transporter. Nat Commun, 5, 5271. https://doi.org/10.1038/ncomms6271

Bayburt, T. H., Carlson, J. W., \& Sligar, S. G. (1998). Reconstitution and imaging of a membrane protein in a nanometer-size phospholipid bilayer. J Struct Biol, 123(1), 37-44. https://doi.org/10.1006/isbi.1998.4007

Bennett, A. E., Griffin, R. G., Ok, J. H., \& Vega, S. (1992). Chemical shift correlation spectroscopy in rotating solids: Radio frequency-driven dipolar recoupling and longitudinal exchange. J. Chem. Phys., 96, 8624-8627.

Bertini, I., Engelke, F., Gonnelli, L., Knott, B., Luchinat, C., Osen, D., \& Ravera, E. (2012). On the use of ultracentrifugal devices for sedimented solute NMR. J Biomol NMR, 54(2), 123-127. https://doi.org/10.1007/s10858-012-9657-y

Clantin, B., Delattre, A. S., Rucktooa, P., Saint, N., Meli, A. C., Locht, C., Jacob-Dubuisson, F., \& Villeret, V. (2007). Structure of the membrane protein FhaC: a member of the Omp85-TpsB transporter superfamily. Science, 317(5840), 957-961. https://doi.org/10.1126/science.1143860

Clore, G. M., \& Iwahara, J. (2009). Theory, practice, and applications of paramagnetic relaxation enhancement for the characterization of transient low-population states of biological macromolecules and their complexes. Chem Rev, 109(9), 4108-4139. https://doi.org/10.1021/cr900033p 
695

Dé, E., Saint, N., Glinel, K., Méli, A. C., Levy, D., \& Jacob-Dubuisson, F. (2008). Influence of the passenger domain of a model autotransporter on the properties of its translocator domain. Mol Membr Biol, 25(3), 192-202. https://doi.org/10.1080/09687680701771925

Delaglio, F., Grzesiek, S., Vuister, G. W., Zhu, G., Pfeifer, J., \& Bax, A. (1995). NMRPipe: a multidimensional spectral processing system based on UNIX pipes. J Biomol NMR, 6(3), 277-293. https://doi.org/10.1007/BF00197809

Delattre, A. S., Clantin, B., Saint, N., Locht, C., Villeret, V., \& Jacob-Dubuisson, F. (2010, Nov). Functional importance of a conserved sequence motif in $\mathrm{FhaC}$, a prototypic member of the TpsB/Omp85 superfamily. FEBS J, 277(22), 4755-4765. https://doi.org/10.1111/i.1742-4658.2010.07881.x

Delattre, A. S., Saint, N., Clantin, B., Willery, E., Lippens, G., Locht, C., Villeret, V., \& Jacob-Dubuisson, F. (2011). Substrate recognition by the POTRA domains of TpsB transporter FhaC. Mol Microbiol, 81(1), 99-112. https://doi.org/10.1111/i.1365-2958.2011.07680.x

Derbise, A., Lesic, B., Dacheux, D., Ghigo, J.-M., \& Carniel, E. (2003). A rapid and simple method for inactivating chromosomal genes in Yersinia. FEMS Immunol Med Microbiol, 38, 113-116. https://doi.org/10.1016/S0928-8244(03)00181-0

Diederichs, K. A., Ni, X., Rollauer, S. E., Botos, I., Tan, X., King, M. S., Kunji, E. R. S., Jiang, J., \& Buchanan, S. K. (2020). Structural insight into mitochondrial beta-barrel outer membrane protein biogenesis. Nat Commun, 11(1), 3290. https://doi.org/10.1038/s41467-020-17144-1

Doerner, P. A., \& Sousa, M. C. (2017). Extreme Dynamics in the BamA beta-Barrel Seam. Biochemistry, 56(24), 3142-3149. https://doi.org/10.1021/acs.biochem.7b00281

Doyle, M. T., \& Bernstein, H. D. (2019). Bacterial outer membrane proteins assemble via asymmetric interactions with the BamA beta-barrel. Nat Commun, 10(1), 3358. https://doi.org/10.1038/s41467-019-11230-9

Estrada Mallarino, L., Fan, E., Odermatt, M., Muller, M., Lin, M., Liang, J., Heinzelmann, M., Fritsche, F., Apell, H. J., \& Welte, W. (2015). TtOmp85, a beta-barrel assembly protein, functions by barrel augmentation. Biochemistry, 54(3), 844-852. https://doi.org/10.1021/bi5011305

Fan, E., Fiedler, S., Jacob-Dubuisson, F., \& Muller, M. (2012). Two-partner secretion of gram-negative bacteria: a single beta-barrel protein enables transport across the outer membrane. J Biol Chem, 287(4), 2591-2599. https://doi.org/10.1074/jbc.M111.293068 
761

762

763

764

765

766

767

768

769

770

771

772

773

774

775

Gu, Y., Li, H., Dong, H., Zeng, Y., Zhang, Z., Paterson, N. G., Stansfeld, P. J., Wang, Z., Zhang, Y., Wang, W., \& Dong, C. (2016). Structural basis of outer membrane protein insertion by the BAM complex. Nature, 531(7592), 64-69. https://doi.org/10.1038/nature17199

Guedin, S., Willery, E., Locht, C., \& Jacob-Dubuisson, F. (1998). Evidence that a globular conformation is not compatible with FhaC-mediated secretion of the Bordetella pertussis filamentous haemagglutinin. Mol Microbiol, 29(3), 763-774. https://doi.org/10.1046/i.13652958.1998.00970.x

Guedin, S., Willery, E., Tommassen, J., Fort, E., Drobecq, H., Locht, C., \& Jacob-Dubuisson, F. (2000). Novel topological features of $\mathrm{FhaC}$, the outer membrane transporter involved in the secretion of the Bordetella pertussis filamentous hemagglutinin. J Biol Chem, 275(39), 30202-30210. https://doi.org/10.1074/jbc.M005515200

Guerin, J., Baud, C., Touati, N., Saint, N., Willery, E., Locht, C., Vezin, H., \& Jacob-Dubuisson, F. (2014). Conformational dynamics of protein transporter FhaC: large-scale motions of plug helix. Mol Microbiol, 92(6), 1164-1176. https://doi.org/10.1111/mmi.12585

Guerin, J., Bigot, S., Schneider, R., Buchanan, S. K., \& Jacob-Dubuisson, F. (2017). Two-partner secretion: Combining efficiency and simplicity in the secretion of large proteins for bacteria-host and bacteria-bacteria interactions. Front Cell Infect Microbiol, 7, 148. https://doi.org/10.3389/fcimb.2017.00148

Guerin, J., Botos, I., Zhang, Z., Lundquist, K., Gumbart, J. C., \& Buchanan, S. K. (2020). Structural insight into toxin secretion by contact-dependent growth inhibition transporters. Elife, 9. https://doi.org/10.7554/eLife.58100

Guerin, J., Saint, N., Baud, C., Meli, A. C., Etienne, E., Locht, C., Vezin, H., \& Jacob-Dubuisson, F. (2015). Dynamic interplay of membrane-proximal POTRA domain and conserved loop L6 in Omp85 transporter FhaC. Mol Microbiol, 98(3), 490-501. https://doi.org/10.1111/mmi.13137

Hagelueken, G., Abdullin, D., \& Schiemann, O. (2015). mtssISuite: Probing biomolecular conformation by spin-labeling Studies. Methods Enzymol, 565, https://doi.org/10.1016/bs.mie.2015.06.006

Hagelueken, G., Ward, R., Naismith, J. H., \& Schiemann, O. (2012, Apr). MtssIWizard: In Silico Spin-Labeling and Generation of Distance Distributions in PyMOL. Appl Magn Reson, 42(3), 377-391. https://doi.org/10.1007/s00723-012-0314-0

Halladin, D. K., Ortega, F. E., Ng, K. M., Footer, M. J., Mitic, N. S., Malkov, S. N., Gopinathan, A., Huang, K. C., \& Theriot, J. A. (2021, Aug). Entropy-driven translocation of disordered proteins through the 
Gram-positive bacterial cell wall. Nat Microbiol, 6(8), 1055-1065. https://doi.org/10.1038/s41564-021-00942-8

Hartmann, J. B., Zahn, M., Burmann, I. M., Bibow, S., \& Hiller, S. (2018). Sequence-specific solution NMR assignments of the beta-barrel insertase BamA to monitor its conformational ensemble at the atomic level. J Am Chem Soc, 140(36), 11252-11260. https://doi.org/10.1021/jacs.8b03220

Heinz, E., \& Lithgow, T. (2014). A comprehensive analysis of the Omp85/TpsB protein superfamily structural diversity, taxonomic occurrence, and evolution. Front Microbiol, 5, 370. https://doi.org/10.3389/fmicb.2014.00370

Höhr, A. I. C., Lindau, C., Wirth, C., Qiu, J., Stroud, D. A., Kutik, S., Guiard, B., Hunte, C., Becker, T., Pfanner, N., \& Wiedemann, N. (2018). Membrane protein insertion through a mitochondrial beta-barrel gate. Science, 359(6373). https://doi.org/10.1126/science.aah6834

ladanza, M. G., Higgins, A. J., Schiffrin, B., Calabrese, A. N., Brockwell, D. J., Ashcroft, A. E., Radford, S. E., \& Ranson, N. A. (2016). Lateral opening in the intact beta-barrel assembly machinery captured by cryo-EM. Nat Commun, 7, 12865. https://doi.org/10.1038/ncomms12865

ladanza, M. G., Schiffrin, B., White, P., Watson, M. A., Horne, J. E., Higgins, A. J., Calabrese, A. N., Brockwell, D. J., Tuma, R., Kalli, A. C., Radford, S. E., \& Ranson, N. A. (2020). Distortion of the bilayer and dynamics of the BAM complex in lipid nanodiscs. Commun Biol, 3(1), 766. https://doi.org/10.1038/s42003-020-01419-w

Iwahara, J., Tang, C., \& Marius Clore, G. (2007). Practical aspects of (1)H transverse paramagnetic relaxation enhancement measurements on macromolecules. J Magn Reson, 184(2), 185-195. https://doi.org/10.1016/j.jmr.2006.10.003

Jeschke, G. (2012). DEER distance measurements on proteins. Annu Rev Phys Chem, 63, 419-446. https://doi.org/10.1146/annurev-physchem-032511-143716

Jeschke, G. (2013). Conformational dynamics and distribution of nitroxide spin labels. Prog Nucl Magn Reson Spectrosc, 72, 42-60. https://doi.org/10.1016/j.pnmrs.2013.03.001

Jeschke, G. (2020). MMM: Integrative ensemble modeling and ensemble analysis. Protein Sci. https://doi.org/10.1002/pro.3965

Jeschke, G., Chechik, V., Ionita, P., Godt, A., Zimmermann, H., Banham, J., Timmel, C. R., Hilger, D., \& Jung, H. (2006). DeerAnalysis - a comprehensive software package for analyzing pulsed ELDOR data 2006, 30, 473-498. Appl Magnetic Reson, 30, 473-498. 
838

839

840

841

842

843

844

845

846

847

848

849

850

851

852

853

854

855

856

857

Knowles, T. J., Scott-Tucker, A., Overduin, M., \& Henderson, I. R. (2009). Membrane protein architects: the role of the BAM complex in outer membrane protein assembly. Nat Rev Microbiol, 7(3), 206214. https://doi.org/10.1038/nrmicro2069

Kurauskas, V., Weber, E., Hessel, A., Ayala, I., Marion, D., \& Schanda, P. (2016). Cross-correlated relaxation of dipolar coupling and chemical-shift anisotropy in magic-angle spinning R1rho NMR measurements: Application to protein backbone dynamics measurements. J Phys Chem B, 120(34), 8905-8913. https://doi.org/10.1021/acs.jpcb.6b06129

Landreh, M., Marty, M., Gault, J., \& Robinson, C. (2016). A sliding selectivity scale for lipid binding to membrane proteins Curr Op Struct Biol, 39, 54-60. https://doi.org/10.1016/j.sbi.2016.04.005

Lee, W., Tonelli, M., \& Markley, J. L. (2015). NMRFAM-SPARKY: enhanced software for biomolecular NMR spectroscopy. Bioinformatics, 31(8), 1325-1327. https://doi.org/10.1093/bioinformatics/btu830

Lewandowski, J. R., Sass, H. J., Grzesiek, S., Blackledge, M., \& Emsley, L. (2011). Site-specific measurement of slow motions in proteins. J Am Chem Soc, 133(42), 16762-16765. https://doi.org/10.1021/ia206815h

Liang, B., \& Tamm, L. K. (2016). NMR as a tool to investigate the structure, dynamics and function of membrane proteins. Nat Struct Mol Biol, 23(6), 468-474. https://doi.org/10.1038/nsmb.3226

Ma, P., Haller, J. D., Zajakala, J., Macek, P., Sivertsen, A. C., Willbold, D., Boisbouvier, J., \& Schanda, P. (2014). Probing transient conformational states of proteins by solid-state $\mathrm{R}(1$ rho) relaxationdispersion NMR spectroscopy. Angew Chem Int Ed Engl, 53(17), 4312-4317. https://doi.org/10.1002/anie.201311275

Maier, T., Clantin, B., Gruss, F., Dewitte, F., Delattre, A. S., Jacob-Dubuisson, F., Hiller, S., \& Villeret, V. (2015). Conserved Omp85 lid-lock structure and substrate recognition in FhaC. Nat Commun, 6, 7452. https://doi.org/10.1038/ncomms8452

Méli, A. C., Hodak, H., Clantin, B., Locht, C., Molle, G., Jacob-Dubuisson, F., \& Saint, N. (2006). Channel properties of TpsB transporter FhaC point to two functional domains with a C-terminal proteinconducting pore. J Biol Chem, 281, 158-166. https://doi.org/10.1074/jbc.M508524200

Mittermaier, A. K., \& Kay, L. E. (2009). Observing biological dynamics at atomic resolution using NMR. Trends Biochem Sci, 34(12), 601-611. https://doi.org/10.1016/i.tibs.2009.07.004

Nadaud, P. S., Helmus, J. J., Hofer, N., \& Jaroniec, C. P. (2007). Long-range structural restraints in spinlabeled proteins probed by solid-state nuclear magnetic resonance spectroscopy. J Am Chem Soc, 129(24), 7502-7503. https://doi.org/10.1021/ja072349t 
862

863

864

865

866

867

868

869

870

871

872

873

874

875

876

877

878

879

880

881

882

883

884

885

886

887

888

889

890

891

892

893

894

895

896

897
Nash, Z. M., \& Cotter, P. A. (2019). Bordetella filamentous hemagglutinin, a model for the Two-Partner $\begin{array}{lllll}\text { Secretion } & \text { pathway. } & \text { Microbiol } & \text { Spectrum, } & \end{array}$ https://doi.org/10.1128/microbiolspec.PSIB-0024-2018

Noinaj, N., Gumbart, J. C., \& Buchanan, S. K. (2017). The beta-barrel assembly machinery in motion. Nat Rev Microbiol, 15(4), 197-204. https://doi.org/10.1038/nrmicro.2016.191

Noinaj, N., Kuszak, A. J., Balusek, C., Gumbart, J. C., \& Buchanan, S. K. (2014). Lateral opening and exit pore formation are required for BamA function. Structure, 22(7), 1055-1062. https://doi.org/10.1016/j.str.2014.05.008

Noinaj, N., Kuszak, A. J., Gumbart, J. C., Lukacik, P., Chang, H., Easley, N. C., Lithgow, T., \& Buchanan, S. K. (2013). Structural insight into the biogenesis of beta-barrel membrane proteins. Nature, 501(7467), 385-390. https://doi.org/10.1038/nature12521

Palmer, A. G., 3rd, \& Massi, F. (2006). Characterization of the dynamics of biomacromolecules using rotating-frame spin relaxation NMR spectroscopy. Chem Rev, 106(5), 1700-1719. https://doi.org/10.1021/cr0404287

Pannier, M., Veit, S., Godt, A., Jeschke, G., \& Spiess, H. W. (2000). Dead-time free measurement of dipoledipole interactions between electron spins. J Magnetic Reson 142, 331-340.

Peterson, J. H., Tian, P., leva, R., Dautin, N., \& Bernstein, H. D. (2010, Oct 12). Secretion of a bacterial virulence factor is driven by the folding of a C-terminal segment. Proc Natl Acad Sci U SA, 107(41), 17739-17744. https://doi.org/10.1073/pnas.1009491107

Renault, M., Bos, M. P., Tommassen, J., \& Baldus, M. (2011). Solid-state NMR on a large multidomain integral membrane protein: the outer membrane protein assembly factor BamA. J Am Chem Soc, 133(12), 4175-4177. https://doi.org/10.1021/ja109469c

Ritchie, T. K., Grinkova, Y. V., Bayburt, T. H., Denisov, I. G., Zolnerciks, J. K., Atkins, W. M., \& Sligar, S. G. (2009). Reconstitution of membrane proteins in phospholipid bilayer nanodiscs. Methods Enzymol, 464, 211-231. https://doi.org/10.1016/S0076-6879(09)64011-8

Ruschak, A. M., \& Kay, L. E. (2010). Methyl groups as probes of supra-molecular structure, dynamics and function. J Biomol NMR, 46(1), 75-87. https://doi.org/10.1007/s10858-009-9376-1

Sahu, I. D., \& Lorigan, G. A. (2020). Electron paramagnetic resonance as a tool for studying membrane proteins. Biomolecules, 10(5). https://doi.org/10.3390/biom10050763 
Schanda, P., \& Brutscher, B. (2005). Very fast two-dimensional NMR spectroscopy for real-time investigation of dynamic events in proteins on the time scale of seconds. J Am Chem Soc, 127(22), 8014-8015. https://doi.org/10.1021/ia051306e

Tian, Y., Han, L., Buckner, A. C., \& Ruotolo, B. T. (2015). Collision induced unfolding of intact antibodies: Rapid characterization of disulfide bonding patterns, glycosylation, and structures. Anal Chem, 87(22), 11509-11515. https://doi.org/10.1021/acs.analchem.5b03291

Tomasek, D., Rawson, S., Lee, J., Wzorek, J. S., Harrison, S. C., Li, Z., \& Kahne, D. (2020). Structure of a nascent membrane protein as it folds on the BAM complex. Nature, 583(7816), 473-478. https://doi.org/10.1038/s41586-020-2370-1

Torricella, F., Pierro, A., Mileo, E., Belle, V., \& Bonucci, A. (2021). Nitroxide spin labels and EPR spectroscopy: A powerful association for protein dynamics studies. Biochim Biophys Acta Proteins Proteom, 1869(7), 140653. https://doi.org/10.1016/j.bbapap.2021.140653

Venditti, V., Fawzi, N. L., \& Clore, G. M. (2011). Automated sequence- and stereo-specific assignment of methyl-labeled proteins by paramagnetic relaxation and methyl-methyl nuclear Overhauser enhancement spectroscopy. J Biomol NMR, 51(3), 319-328. https://doi.org/10.1007/s10858-011$\underline{9559-4}$

Viegas, A., Viennet, T., \& Etzkorn, M. (2016). The power, pitfalls and potential of the nanodisc system for NMR-based studies. Biol Chem, 397(12), 1335-1354. https://doi.org/10.1515/hsz-2016-0224

Vranken, W. F., Boucher, W., Stevens, T. J., Fogh, R. H., Pajon, A., Llinas, M., Ulrich, E. L., Markley, J. L., Ionides, J., \& Laue, E. D. (2005). The CCPN data model for NMR spectroscopy: development of a software pipeline. Proteins, 59(4), 687-696. https://doi.org/10.1002/prot.20449

Warner, L. R., Gatzeva-Topalova, P. Z., Doerner, P. A., Pardi, A., \& Sousa, M. C. (2017). Flexibility in the periplasmic domain of BamA is important for function. Structure, 25(1), 94-106. https://doi.org/10.1016/i.str.2016.11.013

Wu, R., Bakelar, J. W., Lundquist, K., Zhang, Z., Kuo, K. M., Ryoo, D., Pang, Y. T., Sun, C., White, T., Klose, T., Jiang, W., Gumbart, J. C., \& Noinaj, N. (2021, Dec 8). Plasticity within the barrel domain of BamA mediates a hybrid-barrel mechanism by BAM. Nat Commun, 12(1), 7131. https://doi.org/10.1038/s41467-021-27449-4

Xiao, L., Han, L., Li, B., Zhang, M., Zhou, H., Luo, Q., Zhang, X., \& Huang, Y. (2021). Structures of the betabarrel assembly machine recognizing outer membrane protein substrates. FASEB J, 35(1), e21207. https://doi.org/10.1096/fj.202001443RR 
bioRxiv preprint doi: https://doi.org/10.1101/2021.11.09.467682; this version posted January 4,2022 . The copyright holder for this preprint (which was not certified by peer review) is the author/funder, who has granted bioRxiv a license to display the preprint in perpetuity. It is made available under aCC-BY 4.0 International license.

940

941

942

943

944

945

946

947
Zhong, Y., Han, L., \& Ruotolo, B. (2014). Collisional and coulombic unfolding of gas-phase proteins: High correlation to their domain structures in solution Angew. Chem, 53(35), 9209-9212. https://doi.org/10.1002/anie. 201403784

Zhou, D. H., \& Rienstra, C. M. (2008). High-performance solvent suppression for proton detected solidstate NMR. J Magn Reson, 192(1), 167-172. https://doi.org/10.1016/i.jmr.2008.01.012 
bioRxiv preprint doi: https://doi.org/10.1101/2021.11.09.467682; this version posted January 4, 2022. The copyright holder for this preprint (which was not certified by peer review) is the author/funder, who has granted bioRxiv a license to display the preprint in perpetuity. It is made A

B

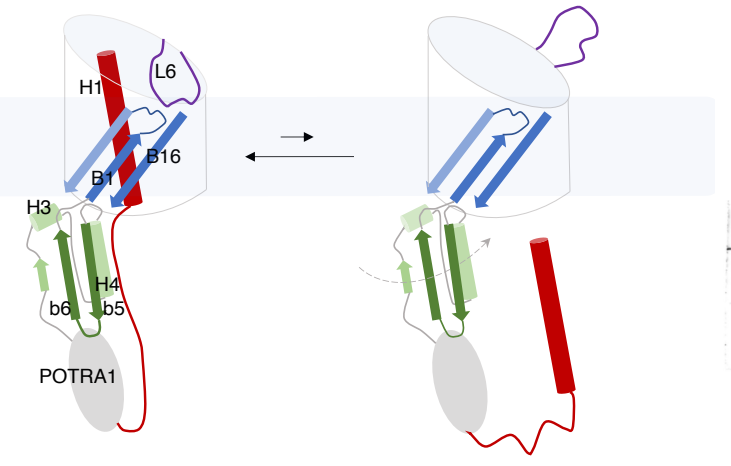

Figure 1. FhaC: Secondary structure and intrinsic dynamics. (A) Linear representation of the secondary structure elements of FhaC, with residues used in this work. L1 to L8 represent the extracellular loops, $\mathrm{b} 1$ to $\mathrm{b} 6$ the $\beta$ strands of the POTRA domains, $\mathrm{H} 1$ to $\mathrm{H} 4$ the $\alpha$ helices, and B1 to B16 the $\beta$-barrel strands. T1 is the first periplasmic turn. (B) Schematic representation of known FhaC motions linked to its activity. Specific structural elements are colored as follows: H1 and the linker in red, the POTRA2 domain in shades of green, L6 in purple, and $\beta$ barrel strands B1, B2 and B16 in shades of blue. In its resting conformation (left), FhaC is closed, with $\mathrm{H} 1$ crossing the $\beta$ barrel. The linker is positioned along the $\mathrm{H} 4$ helix of the POTRA2 domain (Maier et al., 2015), thus obstructing a major substrate binding site (Delattre et al., 2011). L6 is folded inside the barrel with a conserved interaction between its tip and the barrel wall. This conformation is in equilibrium with an open form (right), in which $\mathrm{H} 1$ has vacated the pore and the substrate binding site is available (Guerin et al., 2014). We have obtained evidence that L6 also moves away from its resting position in the $\beta$ barrel and that the POTRA2 domain undergoes so far undefined motions (represented as a dashed line) (Guerin et al., 2015). 
bioRxiv preprint doi: https://doi.org/10.1101/2021.11.09.467682; this version posted January 4, 2022. The copyright holder for this preprint (which was not certified by peer review) is the author/funder, who has granted bioRxiv a license to display the preprint in perpetuity. It is made

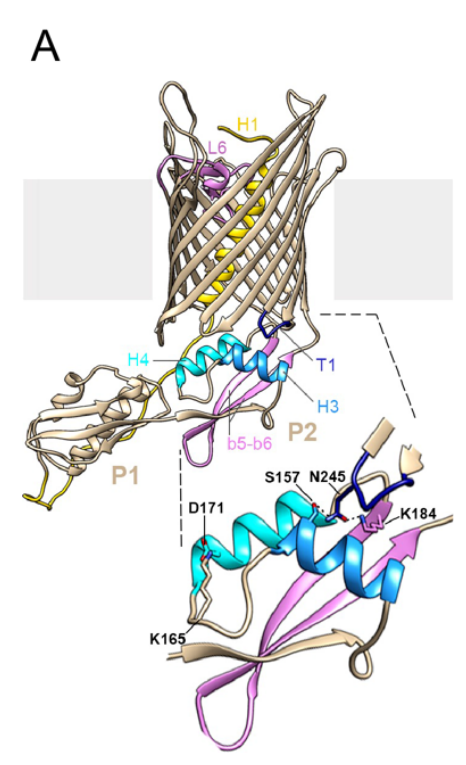
available under aCC-BY 4.0 International license.

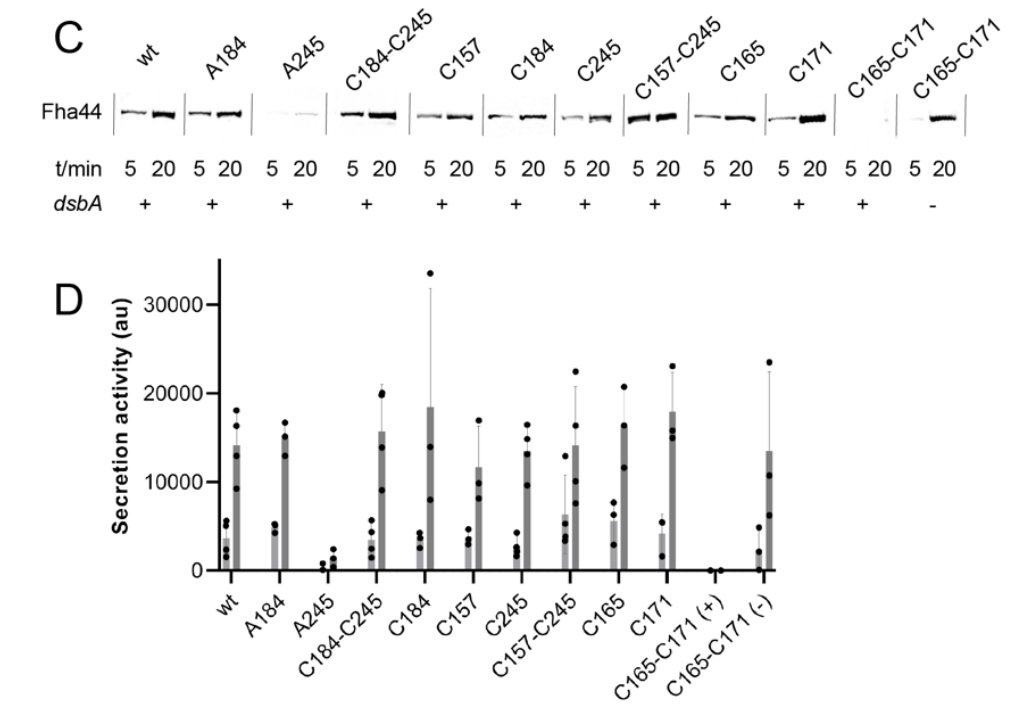

Figure 2. Effects of engineered S-S bonds on FhaC activity. (A) Structural model of FhaC (PDB 4QKY). A zoom of the POTRA2 domain is shown below. (B) Residues involved in a salt bridge $\left(\mathrm{Lys}^{165}-\mathrm{Asp}^{171}\right)$ or $\mathrm{H}$ bonds $\left(\mathrm{Lys}^{184}-\mathrm{Asn}^{245}\right.$; $\left.\mathrm{Ser}^{157}-\mathrm{Asn}^{245}\right)$ in the resting conformation of $\mathrm{FhaC}$ were replaced as indicated $(\mathrm{C}=\mathrm{Cys}$; $\mathrm{A}=\mathrm{Ala})$. Immunoblots were performed on membrane extracts with anti-FhaC antibodies. The asterisks indicate oxidized species of FhaC detected in the absence of the reducing agent dithioerythritol (DTE) in the sample buffer. (C) The secretion activity of the FhaC variants was determined using a model substrate, Fha44-His, affinity precipitated from supernatants 5 and 20 min after induction. Immunoblots were developed with an anti-6His tag monoclonal antibody. (D) Quantification of Fha44 found in culture supernatants after 5 and $20 \mathrm{~min}$. The means and standard deviations of the means are shown ( $\mathrm{n}=3$ or 4$)$. Activity of FhaC ${ }^{\mathrm{C} 165+\mathrm{C} 171}$ could only be detected in the $d s b A \mathrm{KO}$ strain (denoted C165-C171(-)), not in its wild type parent (denoted C165-C171(+)). 
bioRxiv preprint doi: https://doi.org/10.1101/2021.11.09.467682; this version posted January 4, 2022. The copyright holder for this preprint (which was not certified by peer review) is the author/funder, who has granted bioRxiv a license to display the preprint in perpetuity. It is made
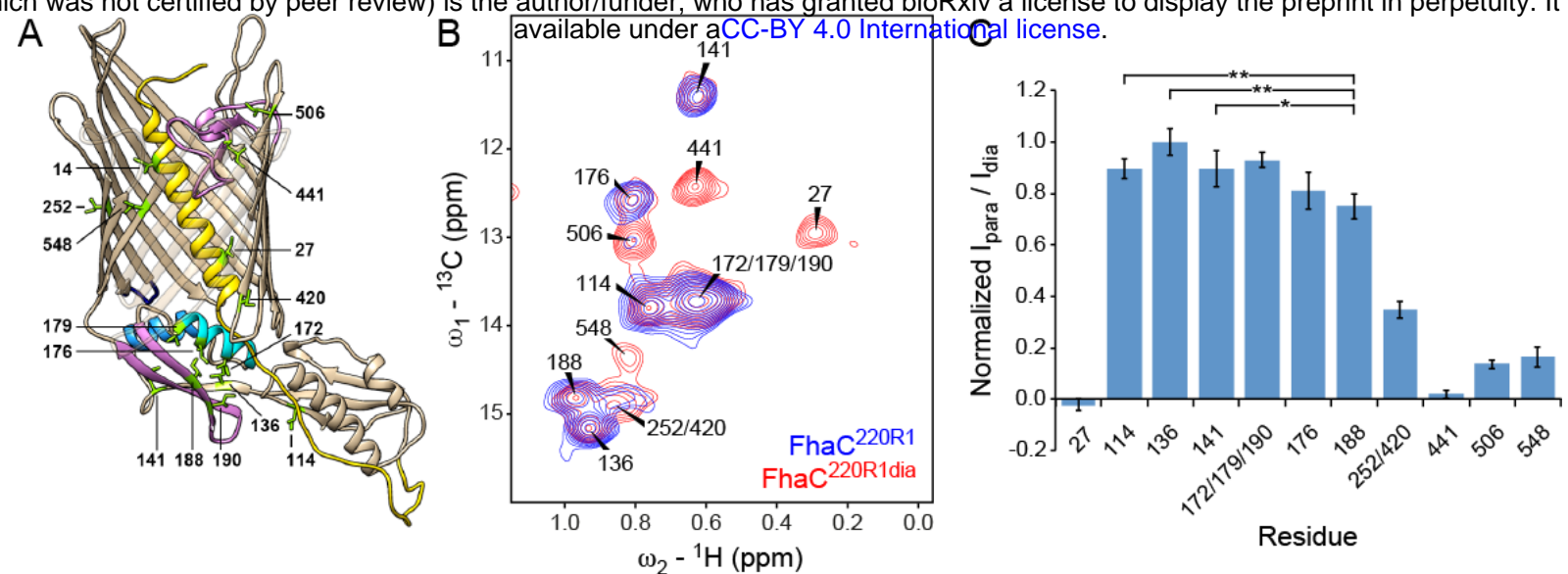

Figure 3. NMR analysis of Ile $\boldsymbol{\delta}_{1}$-methyl labeled FhaC in lipid bilayers. (A) Structure of FhaC with Ile residues labeled and drawn as green sticks. Color code of structural elements is as in Fig. 1. $\beta$-strands 1 to 4 are drawn transparently for visibility. (B) Superposition of the methyl regions of solid-state dipolar $\mathrm{hCH}{ }^{13} \mathrm{C}-{ }^{1} \mathrm{H}$ correlation spectra of $\mathrm{u}-\left({ }^{2} \mathrm{H},{ }^{15} \mathrm{~N}\right)$, Ile$\delta_{1}\left({ }^{13} \mathrm{CH}_{3}\right)$-labeled FhaC samples in E. coli polar lipid liposomes, with a paramagnetic MTSL $\operatorname{tag}\left(\mathrm{FhaC}^{220 \mathrm{R} 1}\right.$, blue) or with a diamagnetic MTSL analog (FhaC ${ }^{220 \mathrm{R} 1 \mathrm{dia}}$, red) attached to the introduced $\mathrm{Cys}^{220}$ residue. Spectra were recorded at $800 \mathrm{MHz}{ }^{1} \mathrm{H}$ Larmor frequency and 50 kHz MAS. (C) Ratios $I_{\text {para }} / I_{\text {dia }}$ of Ile- $\delta 1$ methyl peak intensities in the hCH correlation spectra of FhaC ${ }^{220 \mathrm{R} 1}$ and $\mathrm{FhaC}^{220 \mathrm{R} 1 \mathrm{dia}}$ shown in (B), normalized to the maximum ratio observed in $\mathrm{Ile}^{136}$. Error bars are calculated based on spectral noise levels. * and ** indicate significant ( $\mathrm{p}$ $<0.05$ and $\mathrm{p}<0.01$, respectively) attenuation of the Ile ${ }^{188}$ signal relative to the signals of the reference residues $\mathrm{Ile}^{114}$, Ile ${ }^{136}$, and $\mathrm{Ile}^{141}$. These analyses were complemented by analyses of scalar coupling-based spectra in liposomes and nanodiscs (Supplement 1), through-space correlations (Supplement 2), and relaxation dispersion experiments (Supplement 3). Estimated distances between MTSL probe and Ile residues in $\mathrm{FhaC}^{220 \mathrm{R} 1}$ as expected from the crystal structure are shown in Supplement 4. 

available under aCC-BY 4.0 International license.

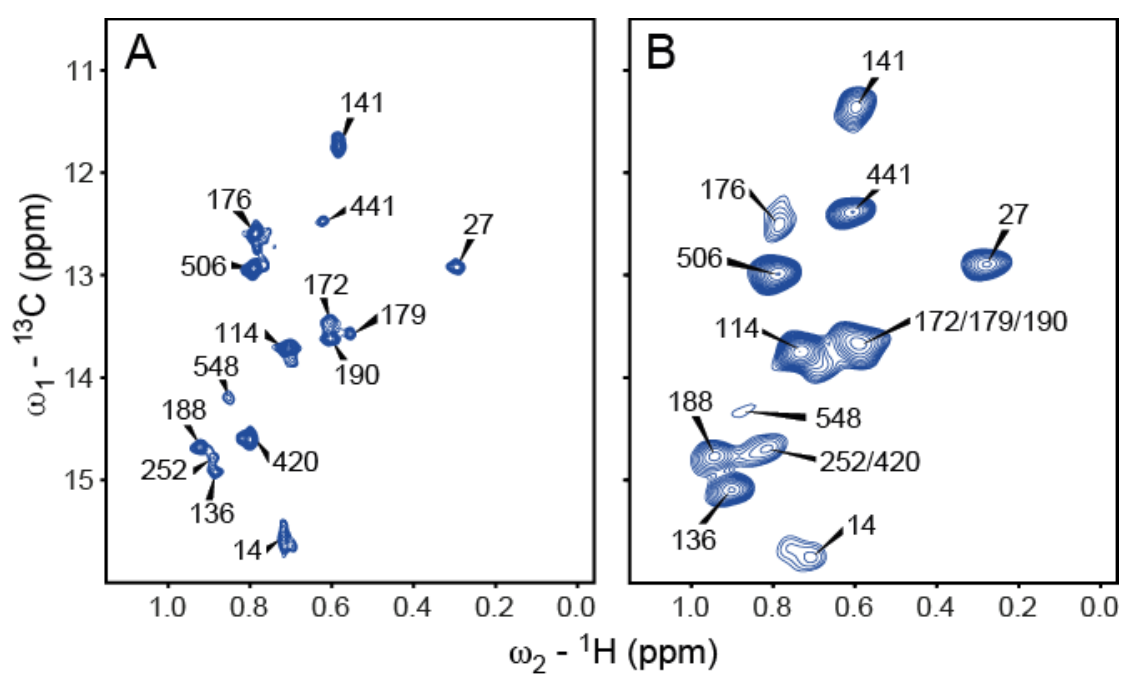

Figure 3 Supplement 1. NMR analyses of Ile $\delta_{1}$ methyl labeled FhaC in lipid bilayers. (A) Methyl region of a solution-state heteronuclear multiple quantum coherence (HMQC) ${ }^{13} \mathrm{C}-{ }^{1} \mathrm{H}$ correlation spectrum of $\mathrm{u}-\left({ }^{2} \mathrm{H},{ }^{15} \mathrm{~N}\right)$, Ile- $\delta_{1}\left({ }^{13} \mathrm{CH}_{3}\right)$-labeled FhaC ${ }^{195 R} 1$ in ${ }^{2} \mathrm{H}-\mathrm{MSP} 1 \mathrm{D} 1$ nanodiscs prepared from deuterated $\left(\mathrm{d}_{54^{-}}\right)$DMPC and DMPG (2:1) lipids, recorded on a 900 MHz spectrometer. The MTSL tag on residue 195 was reduced with ascorbic acid; peak positions are identical to those of wt FhaC in nanodiscs. (B) Same region of a scalar couplingbased solid-state $\mathrm{J}-\mathrm{HSQC}{ }^{13} \mathrm{C}-{ }^{1} \mathrm{H}$ correlation spectrum of wt FhaC (same isotope labeling as in (A)) in d 54 -DMPC/DMPG liposomes, recorded on an $800 \mathrm{MHz}$ spectrometer at $50 \mathrm{kHz}$ MAS.

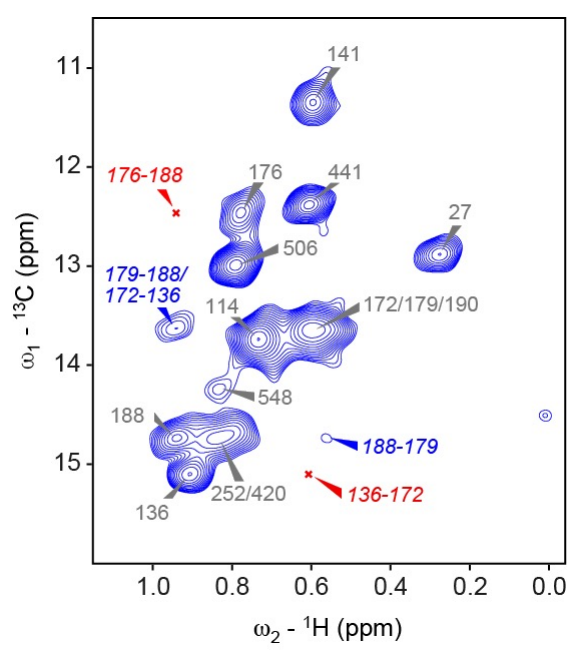

Figure 3 Supplement 2. NMR analysis of through-space contacts between Ile $\delta_{1}$ methyl groups in FhaC in liposomes. 2D hChH correlation spectrum with $6.4 \mathrm{~ms}$ RFDR (Bennett et al., 1992) ${ }^{1} \mathrm{H}-{ }^{1} \mathrm{H}$ mixing of FhaC u- $\left({ }^{2} \mathrm{H},{ }^{15} \mathrm{~N}\right)$, Ile- $\delta_{1}\left({ }^{13} \mathrm{CH}_{3}\right)$ in deuterated $\left(\mathrm{d}_{54}-\right) \mathrm{DMPC} /$ DMPG liposomes, recorded on an $800 \mathrm{MHz}$ NMR spectrometer at $50 \mathrm{kHz}$ MAS, to visualize through-space correlations between Ile $\delta_{1}$ methyl groups close in space. Among expected inter-residue cross-peaks $\left({ }^{1} \mathrm{H}-{ }^{1} \mathrm{H}\right.$ distance below $\left.6 \AA\right)$, peaks present in the spectrum are indicated in blue, those which are absent in red. 
bioRxiv preprint doi: https://doi.org/10.1101/2021.11.09.467682; this version posted January 4, 2022. The copyright holder for this preprint (which was not certified by peer review) is the author/funder, who has granted bioRxiv a license to display the preprint in perpetuity. It is made available under aCC-BY 4.0 International license.
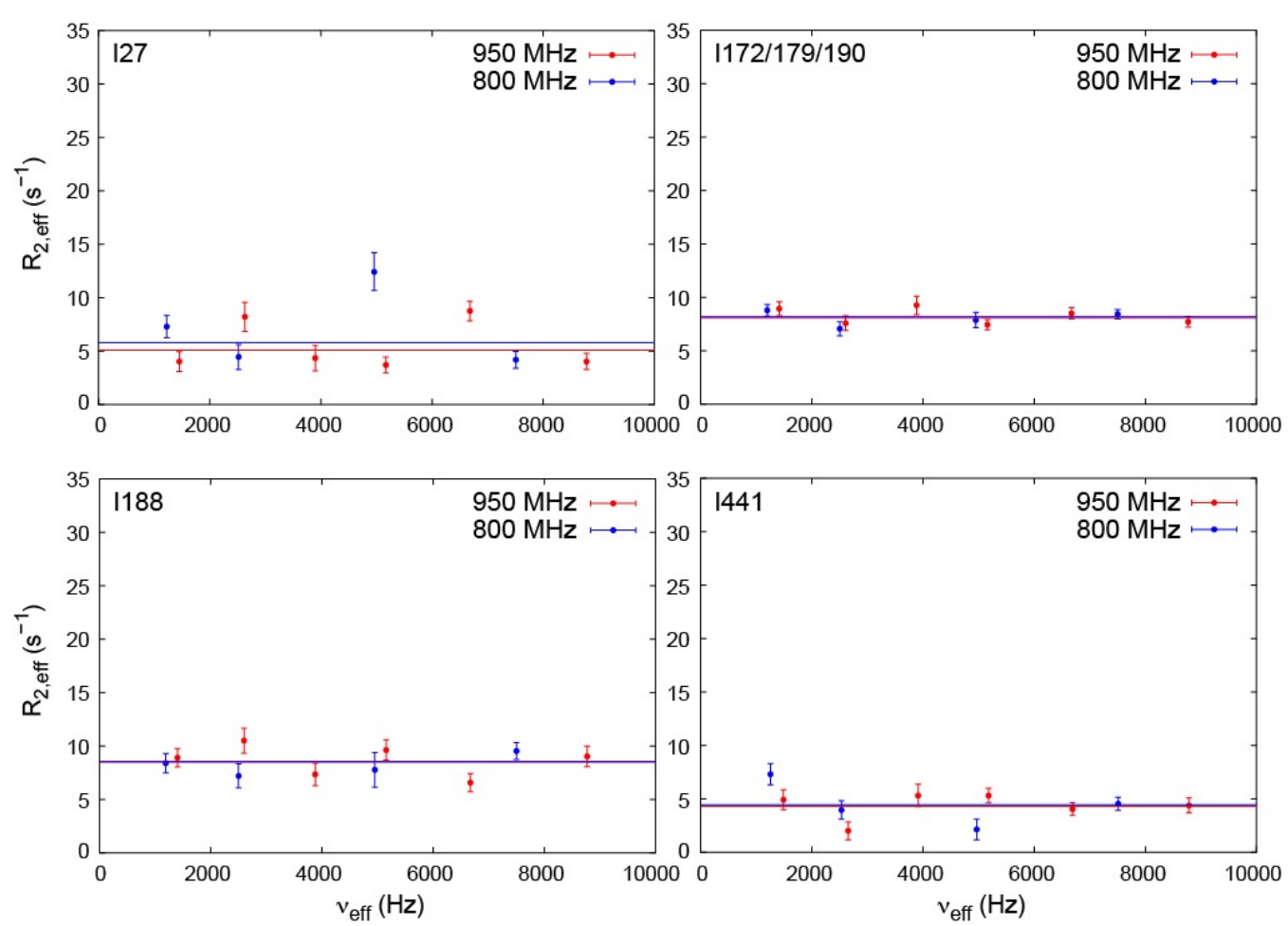

Figure 3 Supplement 3. NMR relaxation dispersion experiments to measure $\mu$ s time scale exchange dynamics in FhaC. Effective ${ }^{13} \mathrm{C}$ transverse relaxation rates $R_{2, \text { eff }}$ extracted from solid-state NMR $R_{1 \rho}$ relaxation dispersion experiments (Lewandowski et al., 2011; Ma et al., 2014) on selected Ile- $\delta_{1}$ methyl groups of $\mathrm{u}-\left({ }^{2} \mathrm{H},{ }^{15} \mathrm{~N}\right)$, Ile- $\delta_{1}\left({ }^{13} \mathrm{CH}_{3}\right)$-labeled wt FhaC, recorded on 800 (blue) and $950 \mathrm{MHz}$ (red) spectrometers at $50 \mathrm{kHz}$ MAS frequency and $17^{\circ} \mathrm{C}$ sample temperature. Horizontal lines are best fits to the data using a model of no exchange (i.e. constant $R_{2, \text { eff }}$ values for varying applied $B_{1}$ radiofrequency fields and thus varying effective fields $\left.v_{\text {eff }}\right)$. Models assuming exchange do not fit the data significantly better according to $\mathrm{F}$ test statistics or Akaike's information criterion (AIC) in any of the Ile$\delta_{1}\left({ }^{13} \mathrm{CH}_{3}\right)$ groups of FhaC. Notably, data from residue Ile ${ }^{548}$ in strand $\beta 16$, at the barrel junction with strand $\beta 1$, could not be reliably analyzed due to low signal-to-noise. 


\begin{tabular}{c|c} 
Ile residue & $\begin{array}{c}\text { Avg. dist. to } \\
\text { paramag. } \\
\text { center }(\AA)\end{array}$ \\
\hline 14 & 7.1 \\
252 & 11.7 \\
548 & 16.5 \\
27 & 17.5 \\
441 & 18.6 \\
506 & 25.3 \\
\hline 420 & 32.0 \\
179 & 32.1 \\
176 & 34.1 \\
188 & 35.4 \\
141 & 38.4 \\
172 & 40.4 \\
136 & 42.0 \\
190 & 43.5 \\
114 & 56.6
\end{tabular}

Figure 3 Supplement 4. Estimated Ile $C \delta_{1}-$ MTSL distances in the crystal structure conformation of FhaC ${ }^{220 R 1}$. Shown are distances (in $\AA$ ) between Ile $C \delta_{1}$ nuclei and the estimated average position of the paramagnetic center in FhaC with a MTSL spin label on residue 220 (FhaC $\left.{ }^{220 \mathrm{R} 1}\right)$. An ensemble of $200 \mathrm{MTSL}$ conformations compatible with labeling on $\mathrm{FhaC}$ residue 220 was calculated using the mtsslSuite web server (Hagelueken et al., 2012; Hagelueken et al., 2015) (http://www.mtsslsuite.isb.ukbonn.de/) and the FhaC crystal structure (PDB 4QKY). The average position of the paramagnetic center (taken as halfway between nitrogen and oxygen atoms of the MTSL nitroxide ring) was calculated from the coordinates of these 200 conformations; distances from that position to Ile $\mathrm{C} \delta_{1}$ nuclei were calculated using PyMOL (The PyMOL Molecular Graphics System. Schrödinger, LLC). A horizontal line in the table indicates the distance from the paramagnetic center up to which attenuation effects on the NMR resonance of the corresponding Ile residue are expected if FhaC assumes a conformation as in the crystal structure. 
bioRxiv preprint doi: https://doi.org/10.1101/2021.11.09.467682; this version posted January 4, 2022. The copyright holder for this preprint (which was not certified by peer review) is the author/funder, who has granted bioRxiv a license to display the preprint in perpetuity. It is made

A
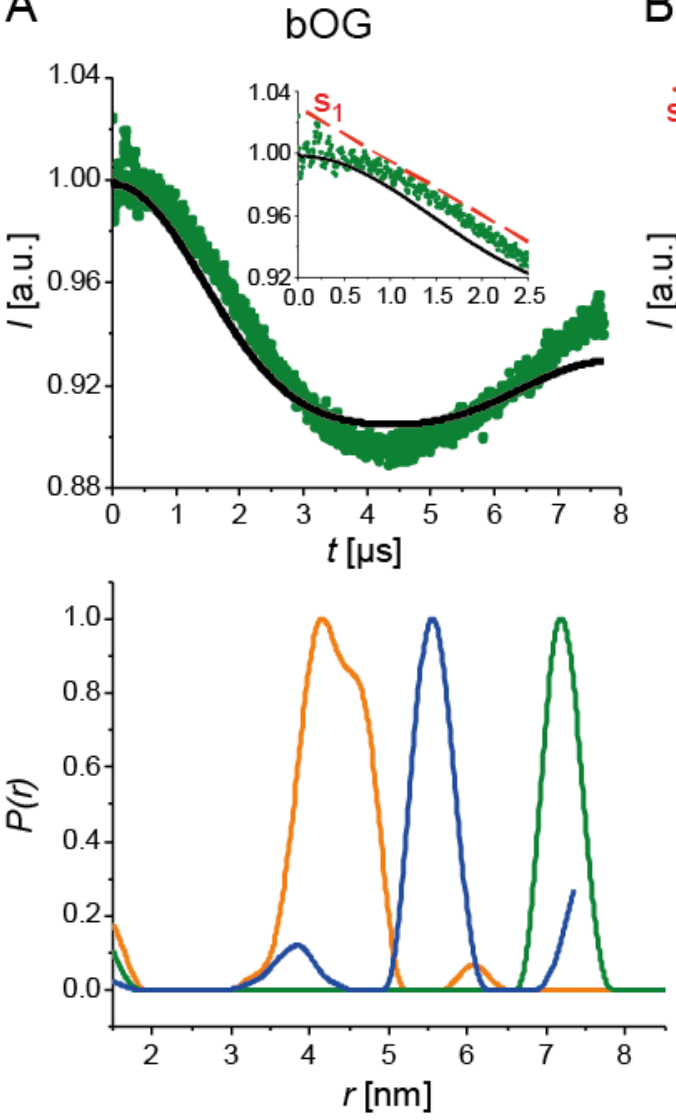

B
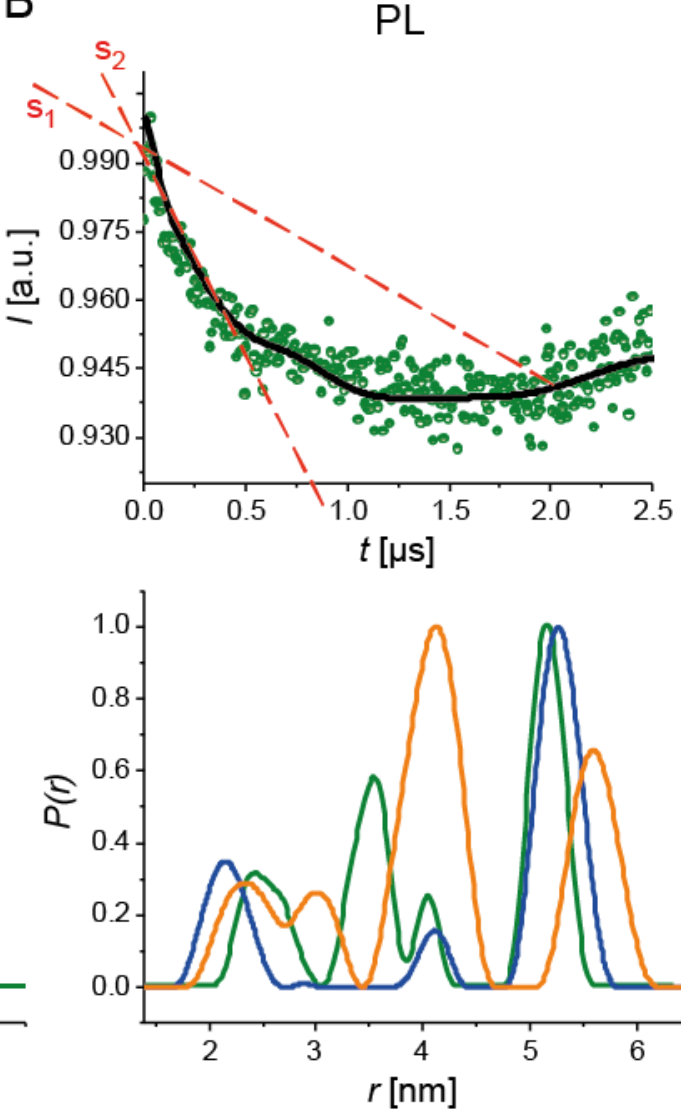

FhaC 33 R1+503R1

FhaC $187 \mathrm{R} 1+503 \mathrm{R} 1$

FhaC ${ }^{195 R 1+503 R 1}$

Figure 4. PELDOR analyses of FhaC. Dipolar evolution function for FhaC ${ }^{195 R 1+503 R 1}$ (top), and distance distributions obtained by Tikhonov regularization of the dipolar evolution functions (bottom) for FhaC ${ }^{33 R 1+503 R 1}$ (orange), FhaC ${ }^{187 R 1+503 R 1}$ (blue), and FhaC ${ }^{195 R 1+503 R 1}$ (green) in bOG (A) and in proteoliposomes (B) prepared with E. coli polar lipids (PL). The black lines in the upper panels correspond to the fitting of the experimental PELDOR traces. The inset in A represents the first $2.5 \mu$ s of the dipolar evolution function for comparison with that shown in (B). The red dashed lines denoted S1 and S2 show the slopes of the first parts of the curves representing the dipolar evolution functions. Predicted distance distributions can be found in Supplement 1 . Note that the longest distances measured depend on the dipolar evolution time $t$. As the lipid environment decreases the $t$ that can be applied, the longest distances shift to smaller values for FhaC in proteoliposomes compared to bOG (Supplement 2). A mutation that severs the connection of the loop L6 to the inner barrel wall affects the EPR spectra of FhaC ${ }^{195 R 1+503 R 1}$ (Supplement 3). Spectroscopic analyses (PELDOR EPR and PRE NMR) of FhaC in nanodiscs complement these data (Supplement 4). 
bioRxiv preprint doi: https://doi.org/10.1101/2021.11.09.467682; this version posted January 4, 2022. The copyright holder for this preprint (which was not certified by peer review) is the author/funder, who has granted bioRxiv a license to display the preprint in perpetuity. It is made available under aCC-BY 4.0 International license.

$33 / 503$

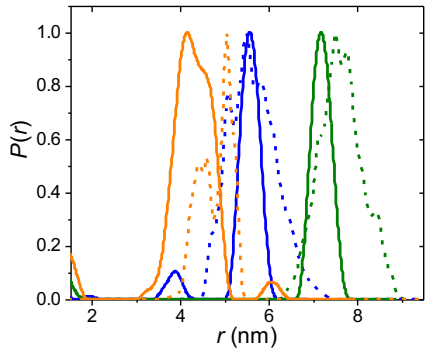

$195 / 503$

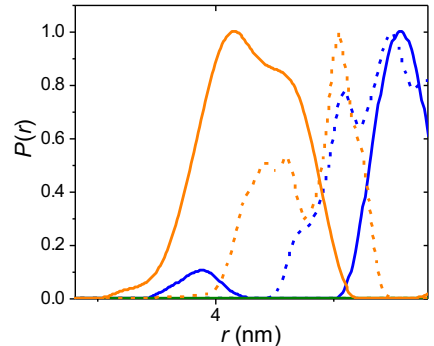

Figure 4 Supplement 1. Distance distributions from PELDOR experiments. The distance distributions obtained for FhaC ${ }^{33 R 1+503 R 1}$ (orange), FhaC ${ }^{187 R 1+503 R 1}$ (blue) and FhaC 195R1+503R1 (green) in bOG (solid lines) are compared with those predicted using a pre-computed rotamer library of the MTSL spin probe attached to specific residues on the PDB structure of FhaC (dashed lines) (Jeschke, 2020). In the right panel, a zoom on the 3-5 $\mathrm{nm}$ region shows the broad distribution for FhaC ${ }^{33 R 1+503 R 1}$.

A
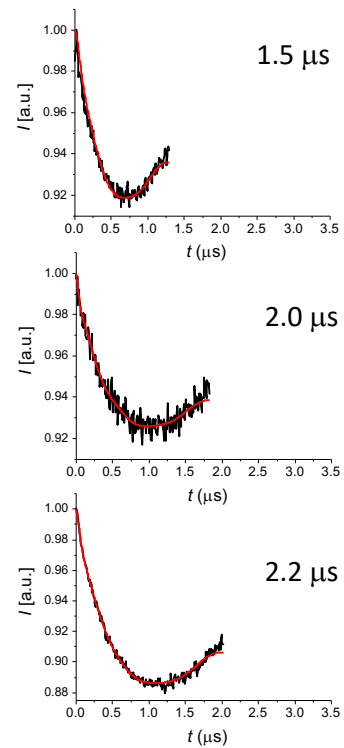
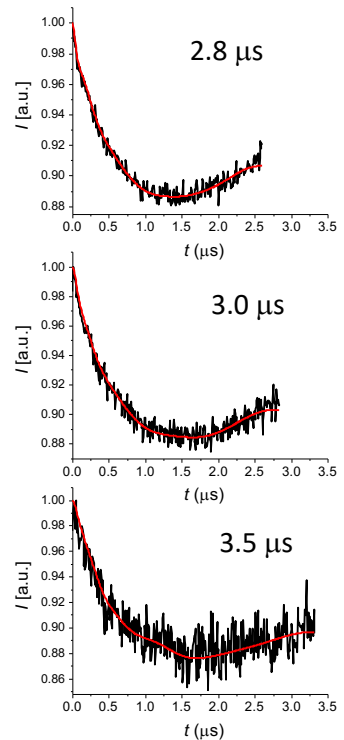

B

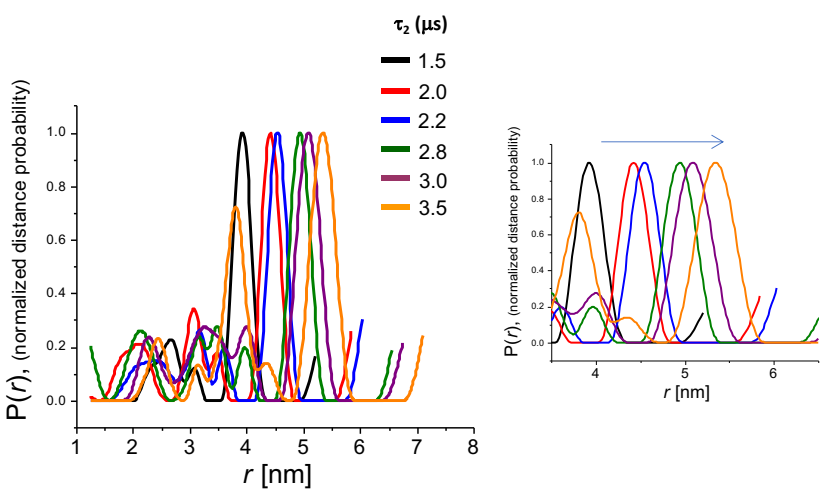

Figure 4 Supplement 2. Dipolar evolution signals recorded with different delays $\tau_{2}$ and corresponding distance distributions. FhaC ${ }^{195 \mathrm{R} 1+503 \mathrm{R} 1}$ in $E$. coli lipids liposomes was used in this experiment. (A) The dipolar evolution signals were measured at increasing dipolar evolution times $t$. (B) The longest distance measured shifts to longer values for longer dipolar evolution times $t$ since long, but not short distances are sensitive to the value used in PELDOR experiments. The lipid environment decreases the dipolar evolution time that can be applied, which results in an apparent shift to smaller distance distribution values. The right panel is a zoom on the 4-6 nm region. 

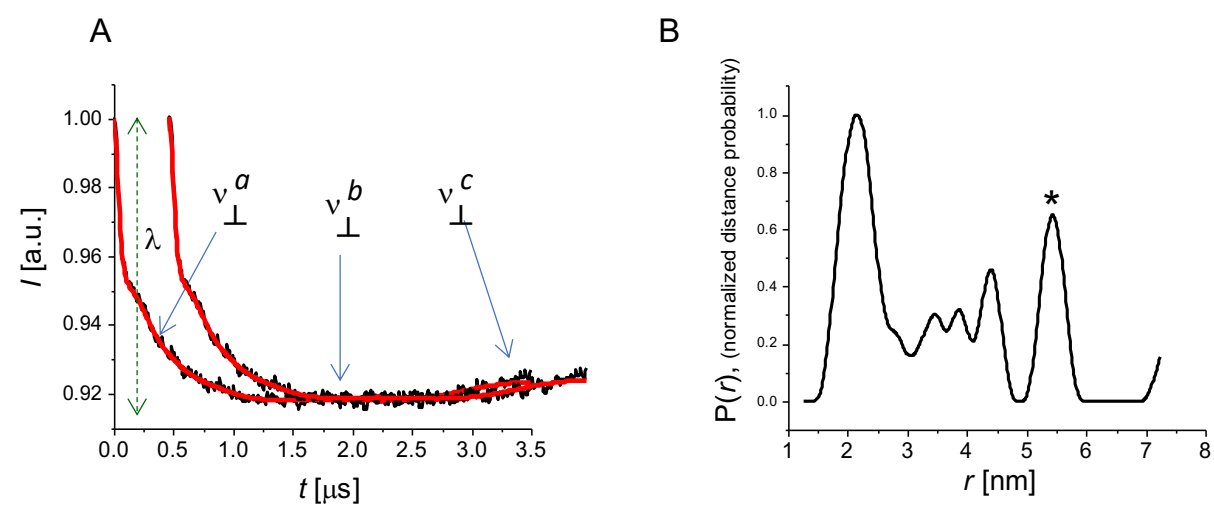

Figure 4 Supplement 3. Effect of the L6-barrel interaction on conformational changes of FhaC. (A) Dipolar evolution function for the PELDOR signal for FhaC ${ }^{\mathrm{R} 492+195 \mathrm{R} 1+503 \mathrm{R} 1}$. (B) Distance distribution obtained by Tikhonov regularization of the signal depicted in (A). The asterisk corresponds to the longest distance measurable as a function of the $t$ parameter applicable in this experiment. 

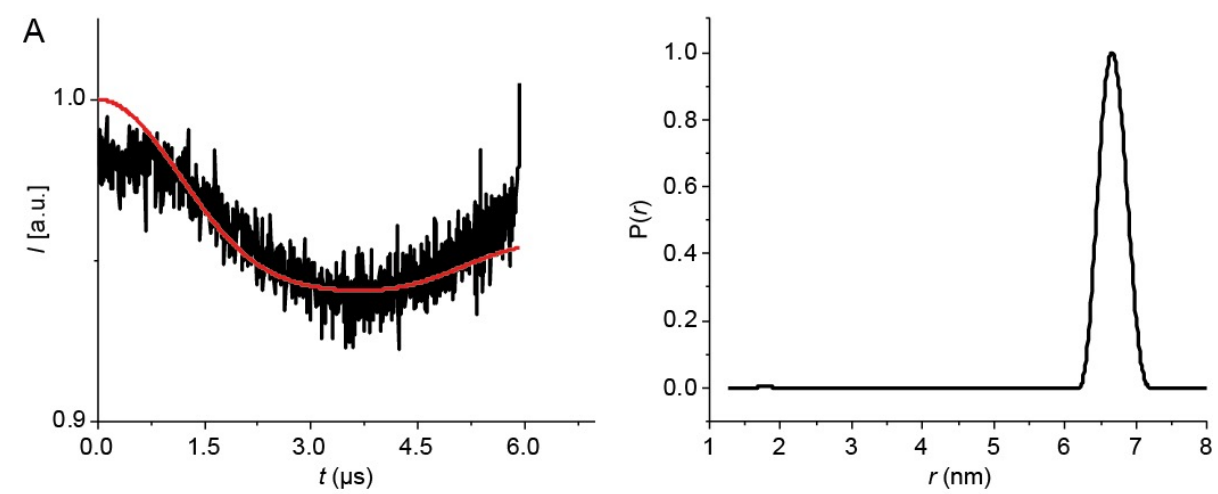

B

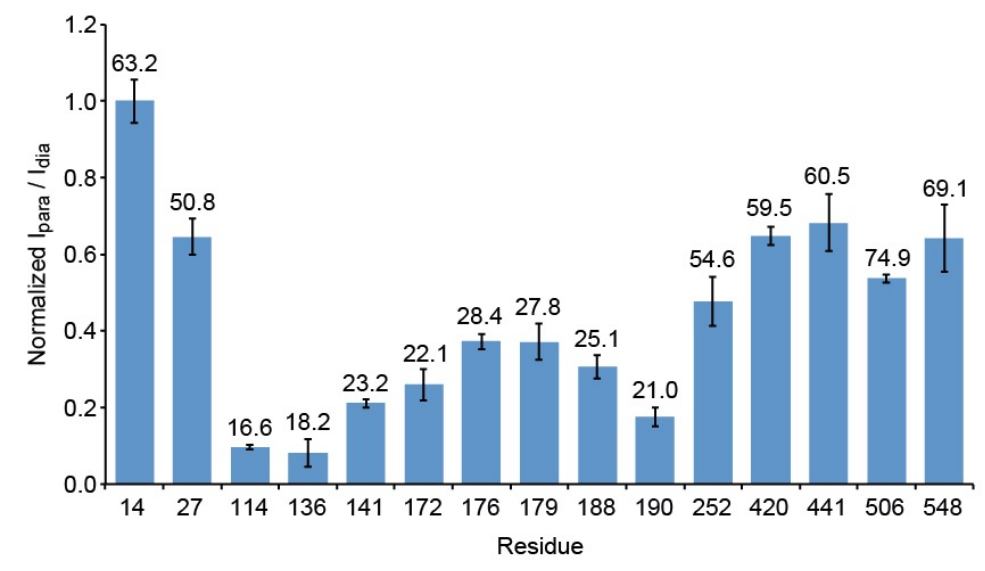

Figure 4 Supplement 4. Spectroscopic analyses of FhaC in nanodiscs. (A) Dipolar evolution function (left) and Tikhonov regularization (right) of the PELDOR signal of FhaC ${ }^{195 R 1+503 R 1}$ in nanodiscs. (B) NMR paramagnetic relaxation enhancement experiments on FhaC ${ }^{195 R 1}$ in nanodiscs. Ratios $I_{\text {para }} / I_{\text {dia }}$ of paramagnetic vs. diamagnetic FhaC ${ }^{195 R 1}$ Ile- $\delta_{1}$ methyl peak intensities (normalized to their maximum value found for Ile ${ }^{14}$; see Methods) extracted from ${ }^{13} \mathrm{C}-{ }^{1} \mathrm{H}$ heteronuclear multiple-quantum coherence (HMQC) experiments in solution before and after reduction of the MTSL spin label by addition of a 10 -fold molar excess of ascorbic acid. Spectra were recorded on a $900 \mathrm{MHz}$ spectrometer at $32^{\circ} \mathrm{C}$. Error bars are calculated based on spectral noise levels. Numbers above the bars indicate the distance between the $\mathrm{C} \delta_{1}$ nucleus of the corresponding Ile residue and the average position of the paramagnetic center in an ensemble of conformations of the MTSL tag attached to Cys ${ }^{195}$ in the FhaC crystal structure, calculated using the MtsslWizard PyMOL plugin (Hagelueken et al., 2012). Relative levels of signal attenuation due to the MTSL tag are perfectly in line with the relative distances of the corresponding residues from the paramagnetic center modeled onto the FhaC crystal structure, suggesting that $\mathrm{FhaC}$ in nanodiscs does not populate alternative conformations. 
A

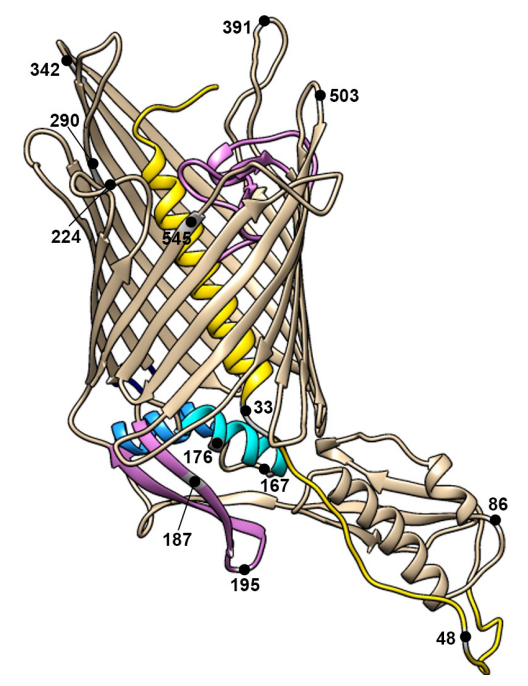

B

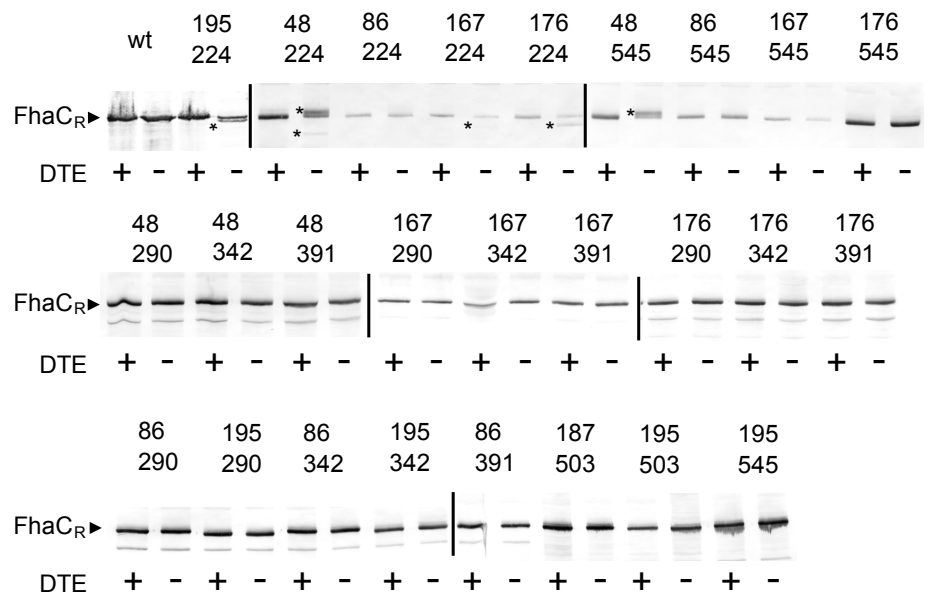

Figure 5. Detection of transient conformers of FhaC in vivo. (A) Position of the Cys substitutions in FhaC. (B) Immunoblot of membrane fractions of E. coli JCB571 (dsbA KO strain) producing FhaC variants. The numbers indicate the positions of the two Cys residues. The reducing agent DTE was added to one half of each sample. $F C_{R} C_{R}$ represents the position of the reduced form. The asterisks point to the additional, cross-linked forms that can migrate faster or more slowly than the reduced form, depending on the respective positions of the two Cys residues. S-S bond formation was confirmed by mass fingerprinting analyses (Figure Supplement 1). 
bioRxiv preprint doi: https://doi.org/10.1101/2021.11.09.467682; this version posted January 4, 2022. The copyright holder for this preprint (which was not certified by peer review) is the author/funder, who has granted bioRxiv a license to display the preprint in perpetuity. It is made available under aCC-BY 4.0 International license.
A
$C^{48}-C^{224}$
1 QAQGSLLPGA RDLNRIDDRQ RKEQIQRDIE RALTRPPVEL NPQSEAAAIC 51 RKPDATSGHT VTVHAVDLDF GVEGRLFDPA PLVQDYLNRP LDNEQLFLLV 101 KALSAALYDR GYATSIVTFV PPGVVDGVLK LKVEWGRIKG WLIDGKPLRG 151 TRDRMMVFSA MPGWQDKVLN VFDIDQATYN INNGGKTGNI TIVPADEYGY 201 SYLDLQLQRR ALPRVSLGMD NSGP@PENG RYKYNASVTA NDLLGLNDTL 251 GLYIGNRYYR DAGHDARRNY DLMYSVPLGR TRLDLQTGYS TYRNLLKTRY 301 GQYQSAGNSR SFGLRATRLL YRDTRSQFSV YGGLRLRQNR NYLAGTRLDV 351 SSRHYSDVTV GMOYSTORGA NAYFGDLSFT RGVGVNNGKY AAYDERGPQG 401 NVSRFNGSLA WTRYMALAGQ PIQWASQLGF QYSRQQLLNS YQITVGDEYT 401 NVSRFNGSLA WTRYMALAGQ PIQWASQLGF QYSRQQLLNS YQITVDEYT 501 NHPDARTIRM AGLAAGVRFD LPYARMSFTY SKPVGAQPGG APRAPVWLYI 551 NAGLSF

\section{B}

1 QAQGSLLPGA RDLNRIDDRQ RKEQLQRDIE RALTRPPVEL NPQSEAAAIO 51 RKPDATSGHT VTVHAVDLDF GVEGRLFDPA PLVQDYLNRP LDNEQLFLLV 101 KALSAALYDR GYATSIVTFV PPGVVDGVLK LRVEWGRIKG WLIDGKPLBG 151 TRDRMMVESA MPGWODKVLN VFDIDQATYN INNGGKTGNI TIVPADEYGY 201 SYLDLQLQRR ALPRVSLGMD NSGPCOPENG RYKYNASVTA NDLLGLNDTL 251 GLYIGNRYYR DAGHDARRNY DLMYSVPLGR TRLDLQTGYS TYRNLLKTRY 301 GQYQSAGNSR SFGLKATRLI YRDTRSQFSV YGGLKLRQNK NYLAGTRLDV 351 SSRHYSDVTV GMQYSTQRGA NAYFGDLSFT RGVGVNNGKY AAYDERGPQG 401 NVSRFNGSLA WTRYMALAGQ PIQWASQLGF QYSRQQLLNS YQITVGDEYT 451 VRGYNLRTSQ SGDSGVYLSN TLTVPVQFSL LGKQASVAPF VGADVGALRS 501 NHPDARTIRM AGLAAGVRFD LPYARMSFTY SKPVGAQPGG APRAPVWLYI 551 NAGLSP
C
$C^{195}-C^{224}$

1 QAQGSLLPGA RDLNRIDDRQ RKEQLQRDIE RALTRPPVEL NPQSEAAAPA 51 RKPDATSGHT VTVHAVDLDF GVEGRLFDPA PLVQDYLNRP LDNEQLFLLV 101 KALSAALYDR GYATSIVTFV PPGVVDGVLK LRVEWGRIRG WLIDGKPLEG 151 TRDRMMVFSA MPGWQDKVLN VFDIDQAIYN INNGGKTGNI TIVPALCGY 201 SYLDLQLQRR ALPRVSLGMD NSGPCOPENG RYKYNASVTA NDLLGLNDTL 51 GLYIGNRYYR DAGHDAERNY DLMYSVPLGR TRLDLQTGYS TYRNLLKTRY 01 GQYQSAGNSR SFGLRATRLL YRDTRSQFSV YGGLKLRONR NYLAGTRLDV 351 SSRHYSDYTV GMOYSTORGA NAYFCDLSET RGYGYNNGKY AAYDERGPOG 401 NVSRFNGSLA WTRYMALAGQ PIQWASQLGF QYSRQQLLNS YQITVGDEYT 451 VRGYNLRTSQ SGDSGVYLSN TLTVPVQFSL LGKQASVAPF VGADVGALRS 501 NHPDARTIRM AGLAAGVRFD LPYARMSFTY SKPVGAQPGG APRAPVWLYI 551 NAGLSE

D

1 QAQGSLLPGA RDLNRIDDRQ RKEQLQRDIE RALTRPPVEL NPQSEAAAPA 51 RRPDATSGHT VTVHAVDLDF GVEGRLFDPA PLVQDYLNRP LDNEQLFLLV 101 KALSAALYDR GYATSTVTFV PPGVYDGYL L LVWWGTRG WLIDGKPLEG

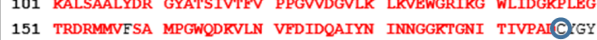
201 SYLDIQIQRR ALPRVSLGMD NSGPCPENG RYKYNASVTA NDLLGLNDTL 251 GLYIGNRYYR DAGHDAERNY DLMYSVPLGR TRLDLQTGYS TYRNLLKTRY 301 GQYQSAGNSR SFGLRATRLL YRDTRSOFSV YGGLKLRQNR NYLAGTRLDV 351 SSKHYSDVTV GMQYSTQRGA NAYFGDLSFT RGVGVNNGKY AAYDERGPOG 401 NVSRFNGSLA WTPYMALAGO PIOWASOLGF OYSRQOLINS YQITVGDEYT 451 VRGYIPTSO SGDSGYY 501 NHPDARTIRM AGLAAGVRPD LPYARMSFTY SKPVGAQPGG APRAPVWLYI 551 NAGLSF

\section{Figure 5 Supplement 1. Peptide mapping analysis of FhaC ${ }^{\mathrm{C} 48-\mathrm{C} 224}$ and FhaC ${ }^{\mathrm{C} 195-C 224}$.}

Residues in red represent sequence coverage with $(\mathrm{A}, \mathrm{C})$ or without $(\mathrm{B}, \mathrm{D})$ reduction and alkylation. In the latter cases, the regions that contain the Cys residues were not characterized, suggesting the presence of an intramolecular S-S bond in both variants. Note that the sequences shown here contain an N-proximal Gly-Ser insertion for cloning purposes that has no effect on the structure or the activity of FhaC. The numbering of FhaC throughout the text corresponds to that of the native protein without this insertion. 
bioRxiv preprint doi: https://doi.org/10.1101/2021.11.09.467682; this version posted January 4, 2022. The copyright holder for this preprint (which was not certified by peer review) is the author/funder, who has granted bioRxiv a license to display the preprint in perpetuity. It is made available unfer aCC-BY 4.0 tnternational license.
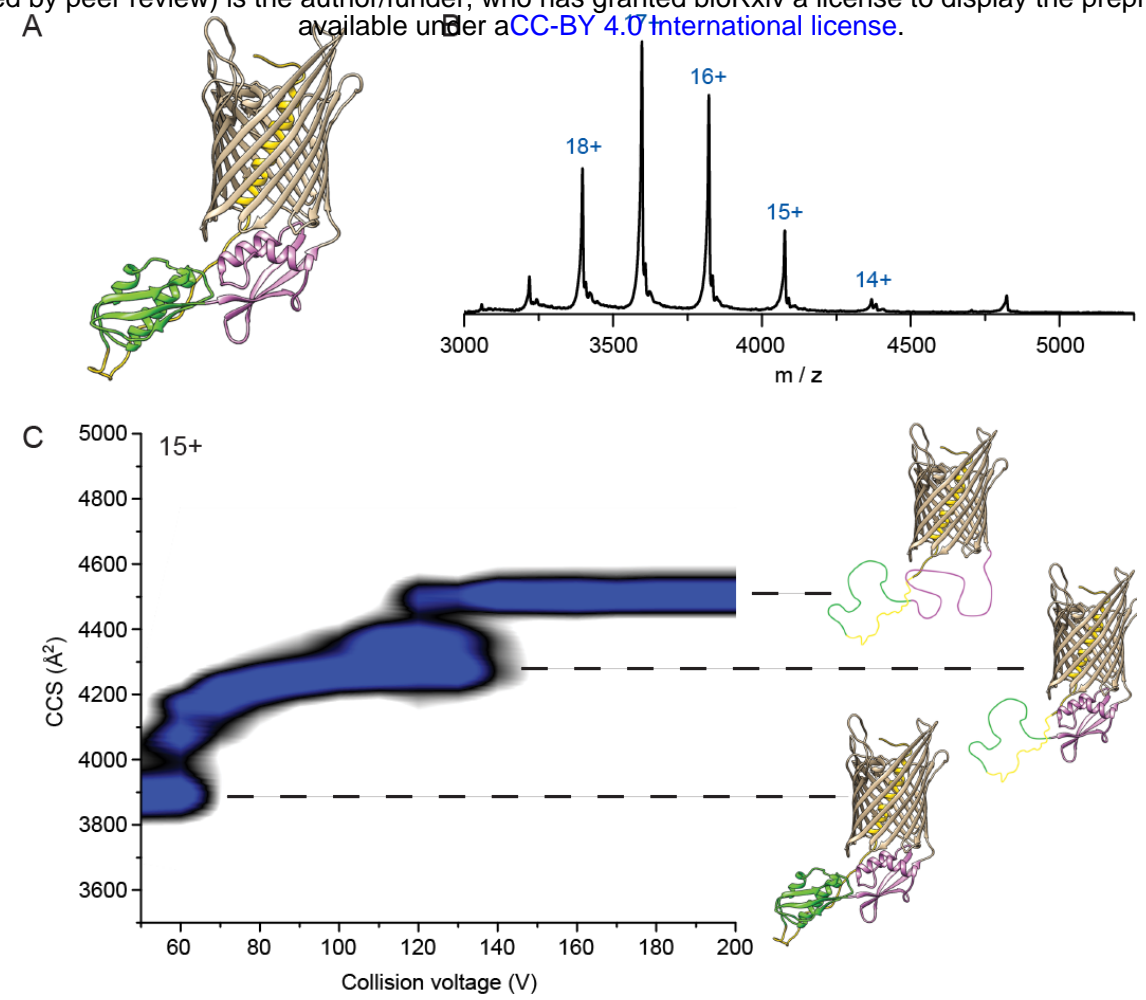

Figure 6. Native mass spectrometry analysis of WT FhaC. (A) Model of FhaC with H1 and the linker in yellow, and the POTRA domains 1 and 2 in pink and green, respectively. (B) Mass spectrum of WT FhaC released from its bOG micelle. The spectra at increasing collision energy are shown in Supplement 1. (C) Collision-induced unfolding (CIU) experiments show two dominant transitions that are likely linked to unfolding of the POTRA domains (see text), although the order in which they unfold is unknown. CIU profiles of control $\beta$-barrel proteins are shown in Supplement 2, and profiles of the FhaC ${ }^{\mathrm{C} 4+\mathrm{C} 391}$ variant with $\mathrm{H} 1$ locked in the barrel are in Supplement 3. 
bioRxiv preprint doi: https://doi.org/10.1101/2021.11.09.467682; this version posted January 4, 2022. The copyright holder for this preprint (which was not certified by peer review) is the author/funder, who has granted bioRxiv a license to display the preprint in perpetuity. It is made available under aCC-BY 4.0 International license.

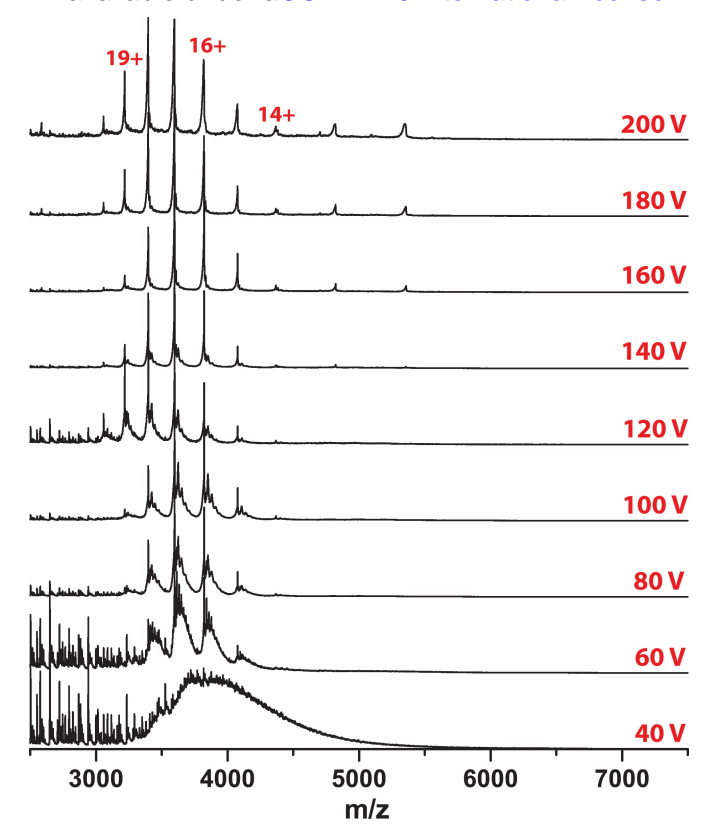

Figure 6 Supplement 1. Native MS analysis of FhaC in bOG micelles. The spectra were obtained at increasing collisional energy. 

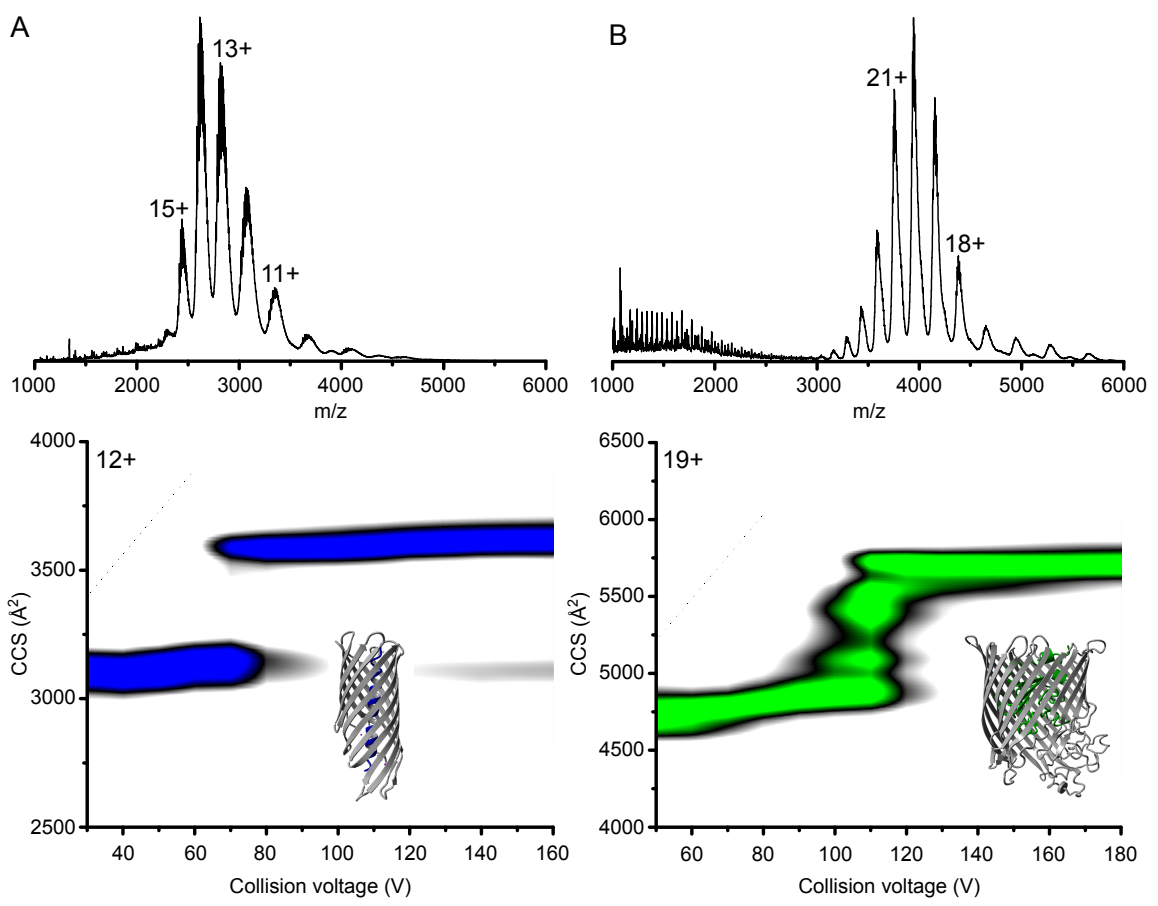

Figure 6 Supplement 2. Mass spectra and CIU plots of control OMPs. (A) SphB1- $\alpha \beta$ is a truncated autotransporter (AT) containing only the $\beta$ barrel with the preceding helical linker inserted in the barrel pore. (B) The TonB-dependent transporter BfrG is composed of a $\beta$ barrel with a soluble N-terminal plug domain inserted in the barrel. The structural models shown are those of related transporters (PDB 1UYN and 3QLB, respectively), as the structures of SphB1- $\alpha \beta$ and BfrG are not available. The mass spectra of the two OMPs released from their bOG micelles are shown at the top, and the CIU plots are below. Both show a single CIU transition, which suggests that the $\beta$ barrels remain intact, while the soluble domains are ejected and unfold. 


\section{A}

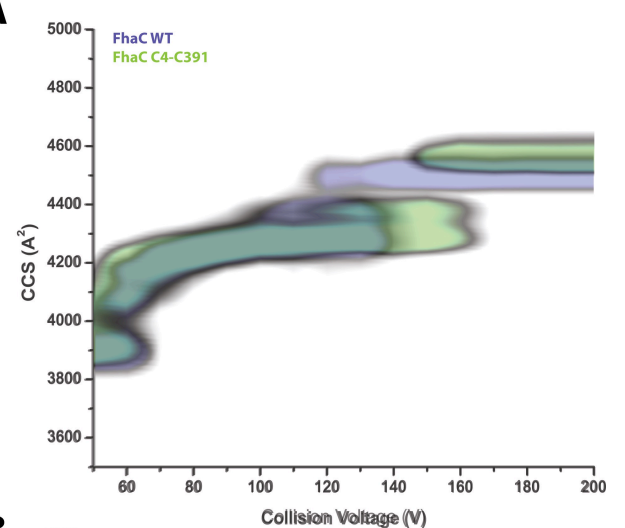

B

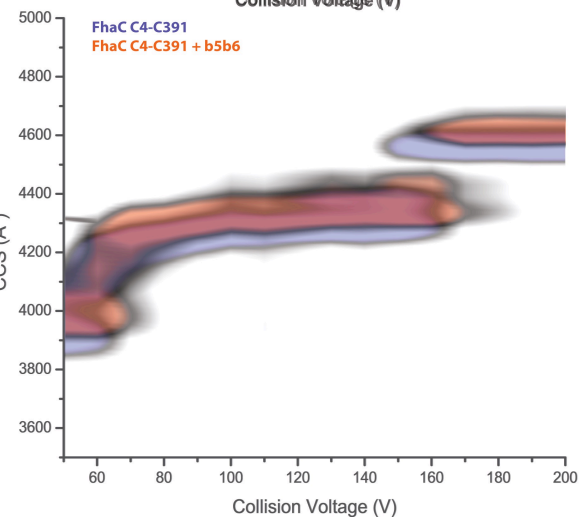

Figure 6 Supplement 3. CIU plots of FhaC ${ }^{\mathbf{C}+\mathbf{C} 391}$. (A) Comparison of the CIU plots of wt FhaC (blue) and the FhaC ${ }^{\mathrm{C} 4+\mathrm{C} 391}$ variant (green). (B) Overlay of the CIU plots of unbound FhaC ${ }^{\mathrm{C} 4+\mathrm{C} 391}$ (blue) and $\mathrm{FhaC}^{\mathrm{C} 4+\mathrm{C} 391}$ with the b5-b6 peptide bound (red). As for wt FhaC (see Figure 7), binding of the peptide to FhaC ${ }^{\mathrm{C} 4+\mathrm{C} 391}$ increased CCS values at both low and high $\mathrm{CE}$, suggesting that it induces enlargement of the $\beta$ barrel, even with $\mathrm{H} 1$ inside. 
bioRxiv preprint doi: https://doi.org/10.1101/2021.11.09.467682; this version posted January 4, 2022. The copyright holder for this preprint (which was not certified by peer review) is the author/funder, who has granted bioRxiv a license to display the preprint in perpetuity. It is made
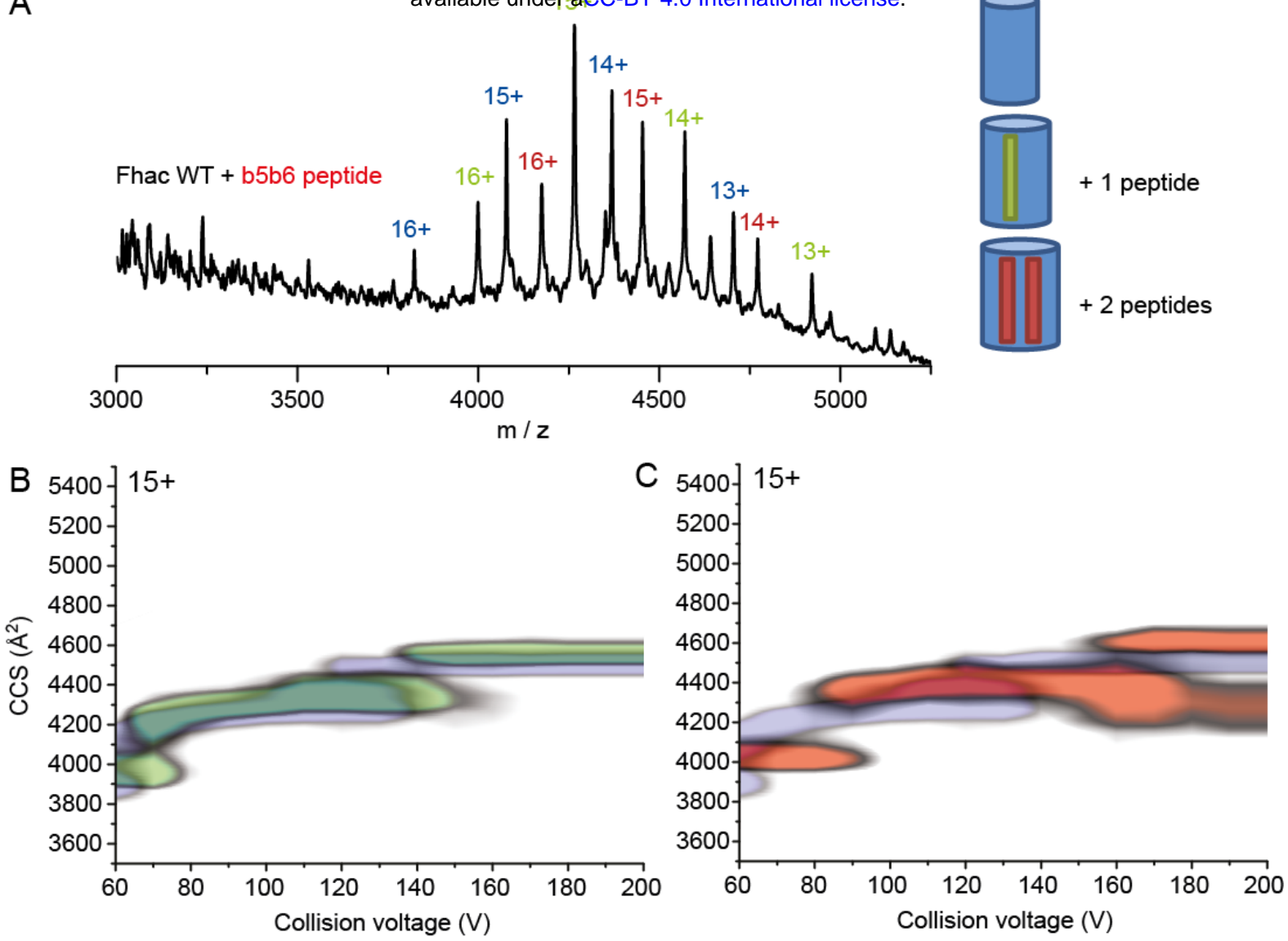

Figure 7. Binding of synthetic peptides to FhaC. (A) Mass spectrum of FhaC incubated with the b5-b6 peptide at a collisional energy of $150 \mathrm{~V}$ shows binding of the peptide to the protein under high-energy conditions. Schematic representations of the barrel with bound peptides are shown at the right. (B) Overlay of the CIU plots of FhaC with (green) and without (blue) one b5-b6 peptide bound. Increased CCS values are observed both under native conditions (low CE) and conditions in which the POTRA domains are most likely unfolded (high CE). (C) Comparison of the CIU plots of FhaC alone (blue) and FhaC with two b5-b6 peptides bound (orange), which shows an additional CCS increase compared to FhaC with a single peptide bound. The peptides used in this study are shown in Supplement 1. Binding of a different peptide and quantification of the binding data are shown in Supplement 2, the CIU plots of wt FhaC with the b4+L and Fha-NT peptides are shown in Supplement 3, and the CCS values measured in those experiments are in Supplement 4. The CIU plot of FhaC ${ }^{\mathrm{C} 4+\mathrm{C} 391}$ with the b5-b6 peptide is shown in Figure 6 Supplement 3. 
bioRxiv preprint doi: https://doi.org/10.1101/2021.11.09.467682; this version posted January 4, 2022. The copyright holder for this preprint (which was not certified by peer review) is the author/funder, who has granted bioRxiv a license to display the preprint in perpetuity. It is made

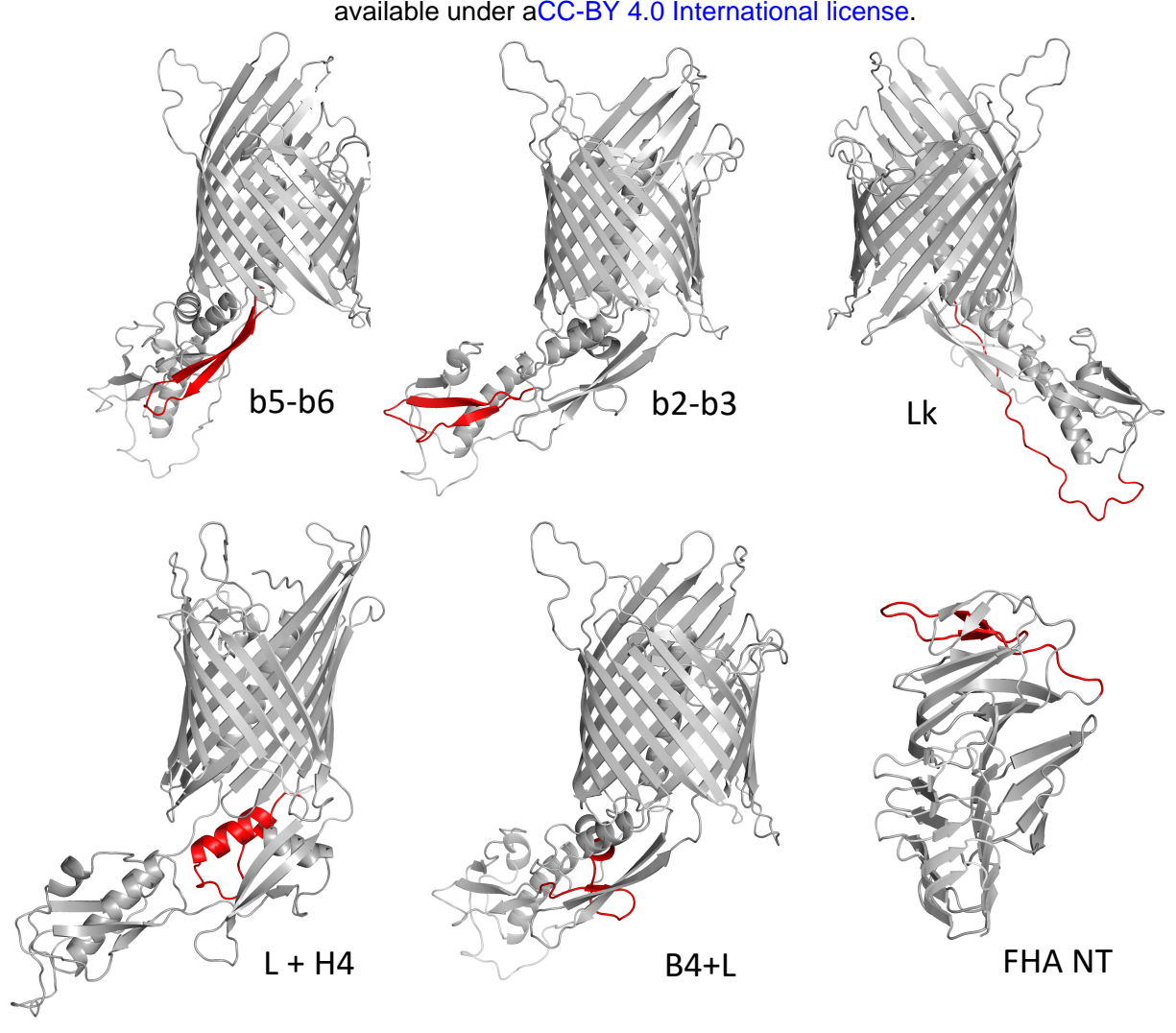

Figure 7 Supplement 1. Synthetic peptides used in this study. The first 5 peptides are shown in red on the structural model of FhaC, and the last one on the structural model of the N-terminal portion of FhaB (PDB 1RWR). The b5-b6 peptide (GKTGNITIVPADEYGYSYLDLQLQR) corresponds to the last two $\beta$ strands of the POTRA2 domain that form an amphipathic $\beta$ hairpin immediately preceding B1, the first strand of the $\beta$ barrel. The b2-b3 peptide (SIVTFVPPGVVDGVLKLKVEWGR) encompasses the last two $\beta$ strands of the POTRA1 domain. The Lk peptide (RPPVELNPQSEAAAPARKPDATSGH) corresponds to the linker between the H1 helix and the POTRA1 domain. The L+H4 peptide (AMPGWQDKVLNVFDIDQAIYNINNG) encompasses the loop (extended) region that precedes the $\mathrm{H} 4 \alpha$ helix and the H4 helix of the POTRA2 domain. The B4+L peptide (RIKGWLIDGKPLEGTRDR) corresponds to the $\beta$ strand b4 of the POTRA2 domain followed by a loop region. Finally, the FHA-NT peptide (QTQVLQGGNKVPVVNIADPNS) corresponds to the N-terminal $\beta$ strands b2 and b3 of FhaB forming a short hairpin, preceded and followed by loop regions. 
bioRxiv preprint doi: https://doi.org/10.1101/2021.11.09.467682; this version posted January 4, 2022. The copyright holder for this preprint (which was not certified by peer review) is the author/funder, who has granted bioRxiv a license to display the preprint in perpetuity. It is made

A


Figure 7 Supplement 2. Binding of synthetic peptides to FhaC. (A) Under similar conditions as in Fig. 7A (collisional energy of $150 \mathrm{~V}$ ), only minimal binding was detected in the mass spectrum of FhaC incubated with the L $+\mathrm{H} 4$ peptide. (B) Quantification of the binding of synthetic peptides to FhaC (light grey) and to the control $\beta$-barrel protein SphB1$\alpha \beta$ (SB1ab, dark grey; used to correct for non-specific binding). Orange bars show normalized values. 


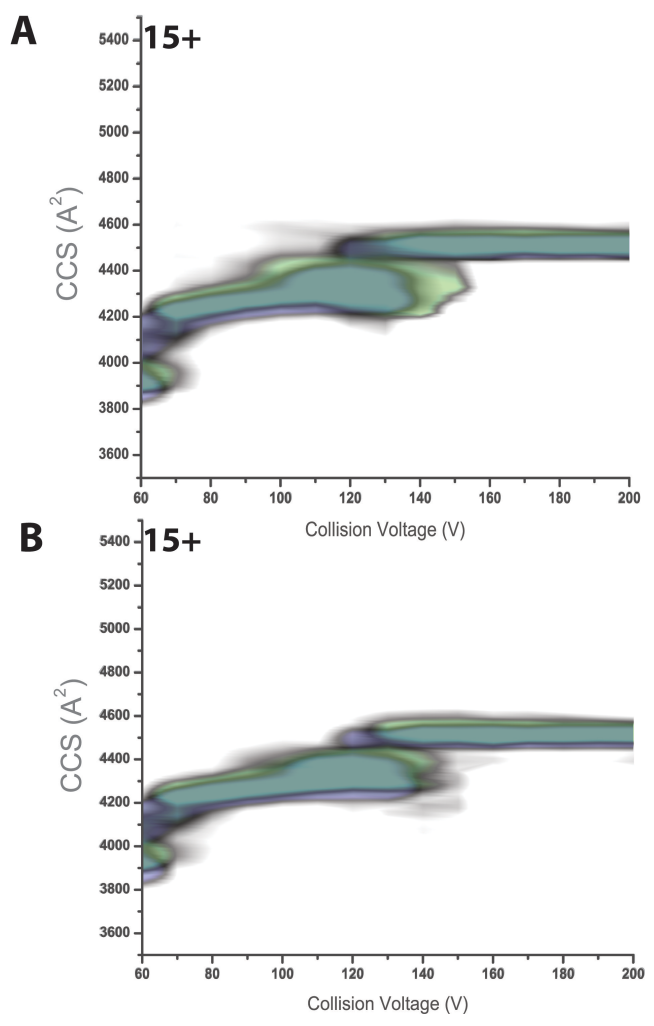

Figure 7 Supplement 3. CIU plots of FhaC incubated with synthetic peptides. (A) FhaC without (blue) or with (green) the Fha-NT peptide. (B) FhaC without (blue) or with (green) the B4+L peptide. In both cases the presence of the peptide caused an increased CCS at low collision voltage, but not at elevated collisional activation.

\begin{tabular}{lccc} 
CCS $\left(\AA^{2}\right)$ & unbound & +1 peptide & +2 peptides \\
\hline FhaC WT & $3863 / 4502$ & - & - \\
+b5b6 & - & $3955 / 4556$ & $4026 / 4595$ \\
+b4L & - & $3954 / 4514$ & - \\
+Fha NT & - & $3954 / 4515$ & - \\
\hline FhaC C4-C391 & $3917 / 4555$ & - & - \\
+b5b6 & - & $3990 / 4607$ & ND/4646
\end{tabular}

Figure 7 Supplement 4. CCS of FhaC with various peptides determined at low and high $\mathrm{CE}$ (listed before and after the slash). The measured values indicate that only b5-b6 enlarges FhaC in both conditions. 


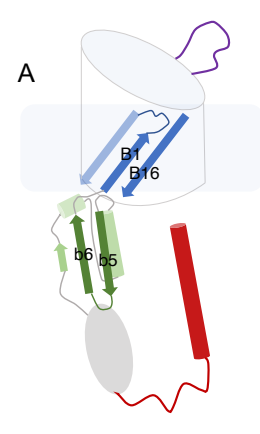

$B$
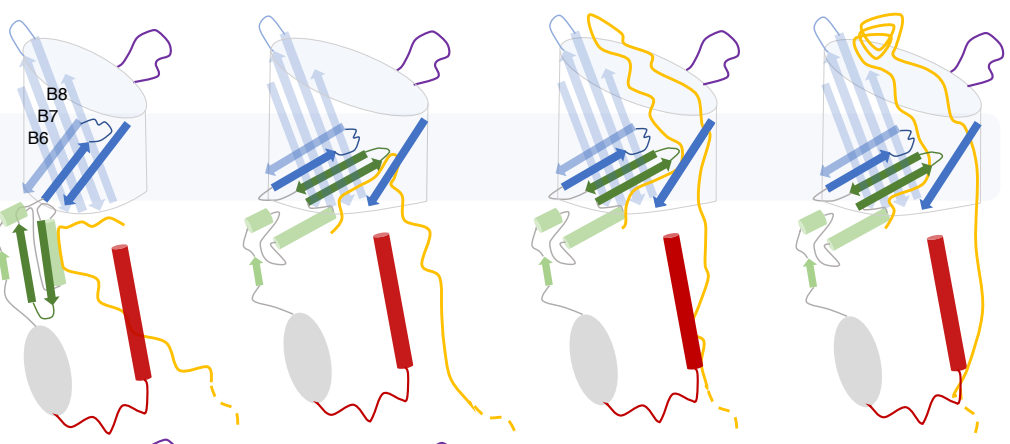

C

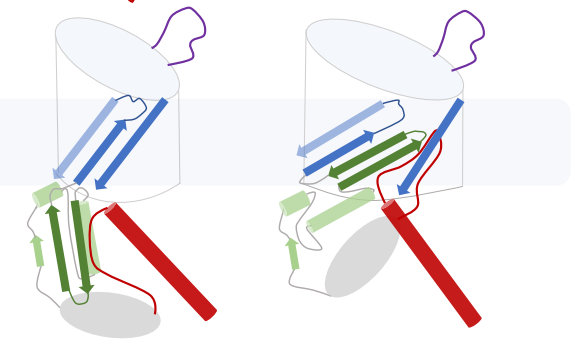

Figure 8. Model for TPS secretion. In the open conformation of FhaC (A), the substrate binding groove between $\mathrm{H} 4$ and $\mathrm{b} 5$ in the POTRA2 domain is available. (B) A specific region of the TPS domain of the substrate (yellow) binds to the POTRA 2 domain, likely by $\beta$ augmentation of the $b 5$ strand. Unzipping of the barrel seam and insertion of the b5-b6 hairpin hoist a first portion of the substrate into the channel. The substrate likely forms a hairpin inside the barrel. Its diffusion toward the surface enables a specific portion of the TPS domain to interact with the B5-B8 beta sheet that protrudes at the cell surface. This interaction templates the folding of the substrate into a nascent $\beta$ helix at the cell surface. The substrate is progressively threaded through the channel and folds at the surface, until a stable nucleus has formed. (C) In the absence of the substrate the linker binds to the POTRA2 domain, and the conformational changes of the protein can hoist it towards the cell surface in a futile cycle. 